Check for updates

Cite this: RSC Adv., 2020, 10, 32581

Received 13th July 2020

Accepted 22nd August 2020

DOI: $10.1039 / \mathrm{dOra06101h}$

rsc.li/rsc-advances

\title{
Regio- and stereoselective intermolecular carbolithiation reactions
}

\author{
G. Marsico, (D) P. Scafato, (D) S. Belviso (D) and S. Superchi (D)*
}

The inter-molecular carbolithiation of unfunctionalized and functionalized alkenes, followed by electrophile trapping, provides a versatile and useful tool for the construction of complex molecular systems, allowing the simultaneous creation of up to two novel carbon-carbon or carbon-heteroatom bonds. In this review a full overview on inter-molecular carbolithiation processes is reported, with a main focus on the chemo-, regio- and stereo-control of the reaction and on the strategies for asymmetric processes.

\section{Introduction}

Since the discovery of organomagnesiums by Barbier ${ }^{1}$ and Grignard, ${ }^{2}$ organometallics have provided very versatile reagents for the carbon-carbon bond formation. Among the several reactions of organometallic nucleophiles with polar carbon electrophiles the carbometalation reaction, i.e. the addition of organometallic reagents to a $\mathrm{C}-\mathrm{C}$ double or triple bond, is particularly appealing, leading to the simultaneous formation of a new $\mathrm{C}-\mathrm{C}$ bond and a new organometallic compound which may give rise to further synthetic transformations. Moreover, if the carbometallation is performed on a 1,2-disubstituted double bond, two regioisomers can be

Dipartimento di Scienze, Università della Basilicata, Via dell'Ateneo Lucano 10, 85100, Potenza, Italy. E-mail: stefano.superchi@unibas.it; Fax: +39-971-205503; Tel: +39971-206098

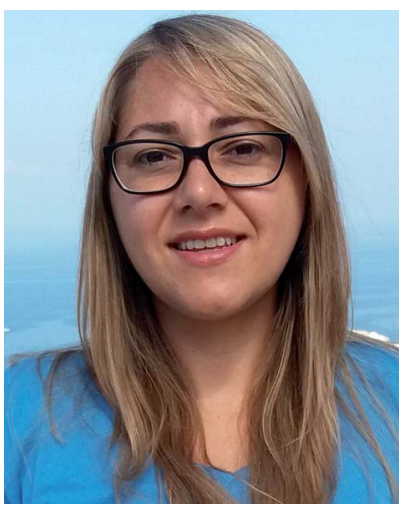

Giulia Marsico received the BSc in Chemistry from University of Basilicata and the PhD degree from University of Salerno under the supervision of Professor Stefano Superchi. She has been visiting PhD student in Jonathan Clayden's group at University of Bristol (UK) working on the synthesis of cyclic oligoureas endowed with cyclochirality. Her research activity focuses on the total synthesis of chiral natural products, on carbolithiations of functionalized alkenes, and on the absolute configuration assignment of chiral molecules by circular dichroism spectroscopy. obtained and, after reaction with a suitable electrophile, two contiguous stereogenic centers can be created (Scheme 1). ${ }^{3,4}$

A subset of the broader family of carbometalations is carbolithiation, i.e. the addition of organolithiums to unsaturated compounds. This reaction displays considerable interest in synthetic organic chemistry, providing an attractive pathway for the efficient construction of new $\mathrm{C}-\mathrm{C}$ bonds by addition of an organolithium reagent to non-activated alkenes or alkynes, also offering the possibility of introducing further functionalization on the molecule by trapping the reactive organolithium intermediates with electrophiles (Scheme 2). ${ }^{\mathbf{5 , 6}}$ Such process is

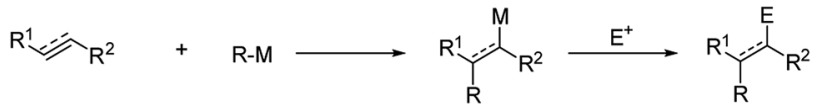

Scheme 1 Carbometalation of unsaturated substrates.

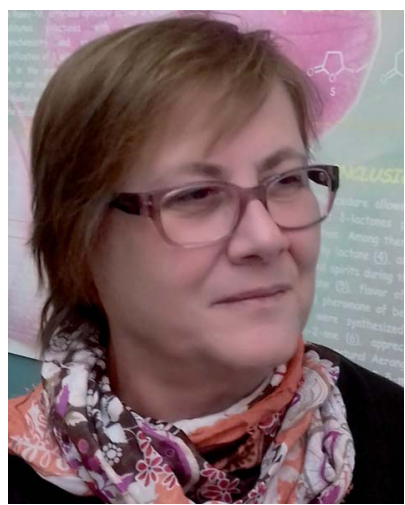

Patrizia Scafato received her Chemistry degree from University of Naples "Federico II" under the supervision of Professor Maria Liliana Graziano, then she moved to University of Basilicata, where she is currently Assistant Professor of Organic Chemistry. She is co-author of more than 55 publications including some reviews. Her research activity focuses on the synthesis of polycyclic heteroaromatics, on the development of chiral catalysts for asymmetric synthesis, on the total synthesis of chiral bioactive natural products. 

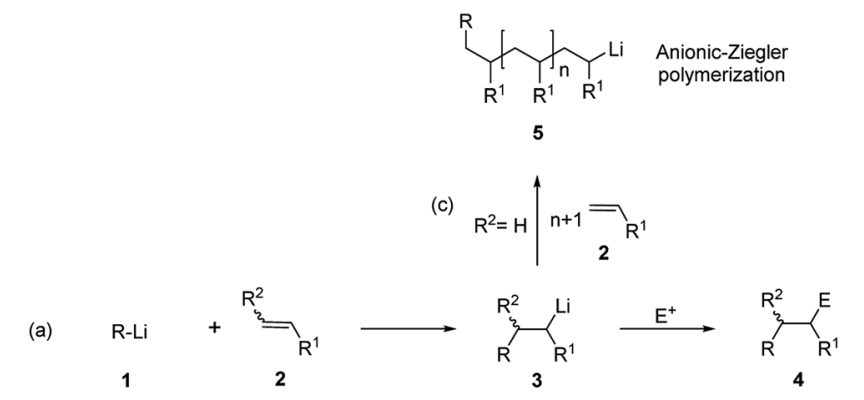

(b)

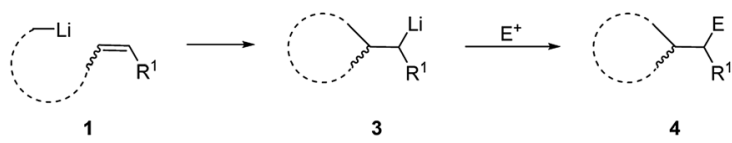

Scheme 2 Carbolithiation-electrophile trapping of alkenes. (a) Intermolecular carbolithiation; (b) intra-molecular carbolithiation; (c) anionic polymerization.

thermodynamically driven and proceeds if the newly formed organolithium is more stable than the starting organometallic reagent. This is the reason why alkyllithiums are much more reactive than the more stable and poorly reactive aryl, vinyl, and methyl lithium derivatives. ${ }^{7}$ As inferred from Scheme 2, carbolithiation can be either an inter-molecular process (route a), giving rise to a chain elongation or an intra-molecular one (route b), providing ring-closing. The most severe limitation to the application of carbolithiation as synthetic method resides in the difficulty to control the high reactivity of the organolithium intermediate towards the unsaturated starting substrate. Indeed, the lithiated intermediate can react with a second molecule of the olefinic substrate, triggering an anionic polymerization process (Scheme 2, route c). In fact, the intermolecular nucleophilic addition of alkyllithium to an alkene represents the first step in anionic polymerization and $n$-BuLi is (a)

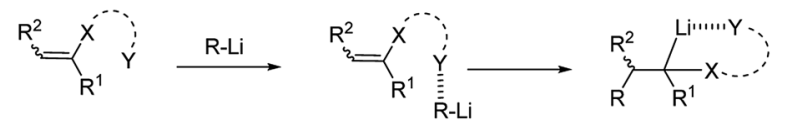

$X, Y=N, O$

(b)

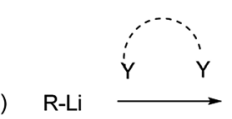

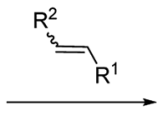

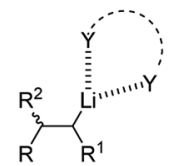

Scheme 3 Heteroatom intra-molecular (route a) and inter-molecular (route b) stabilization of alkyllithiums.

commonly used as catalyst to start the polymerization of styrene, butadiene and isoprene. ${ }^{8}$ It follows that, to achieve a synthetically useful carbolithiation, reaction conditions are required to facilitate the initial carbolithiation, but hampering further addition by the generated organolithium. This can be achieved if the new organometallic $\mathbf{3}$ produced in the addition is less reactive than the starting organolithium 1 (Scheme 2) ) $^{3,9,10}$ and can be facilitated by a stabilization of the organolithium intermediate 3 by intra- or inter-molecular coordination.

Intra-molecular coordination can occur in the presence of groups bearing heteroatom Lewis-base moieties proximal to the reacting alkene (Scheme 3a). They can give rise to both precoordination of the alkyllithium with the alkene and stabilization of the anionic organolithium resulting after carbolithiation. The former pre-complexation step, also termed ComplexInduced Proximity Effect (CIPE), is often invoked to explain the formation of unexpected kinetic products, instead of the apparently available thermodynamic alternatives, and the dramatic acceleration of normally unfavorable reactions. ${ }^{11} \mathrm{~A}$ representative example of application of the CIPE concept is provided by the organolithium-mediated deprotonations like aromatic directed ortho-metalation (DoM) methodologies. ${ }^{12}$

Inter-molecular coordination (Scheme $3 \mathrm{~b}$ ) is instead promoted by coordinating solvents or additives, like tertiary

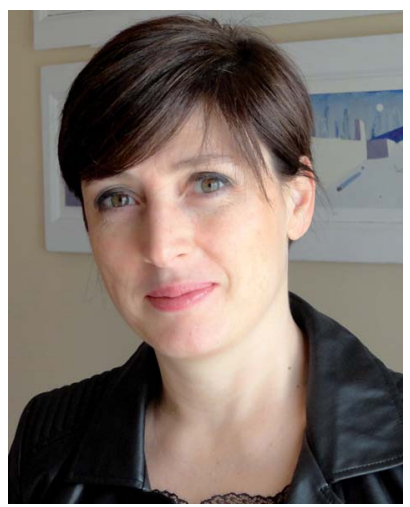

Sandra Belviso got her BSc in Chemistry from University of Basilicata and the PhD degree from University of Salerno. She also spent a short period at the University of St. Andrews (Scotland). She was then appointed Assistant Professor of Inorganic Chemistry at the University of Basilicata where she is supervisor of the "Inorganic Chemistry Laboratory" of the Science Department. Her research interests focus on the synthesis and characterization of metal complexes and tetrapyrrole macrocycles suitable for liquid-crystal, optoelectronic, and organic photovoltaic materials, as well as on the synthesis of zeolite-incapsulated tetrapyrroles for environmental, material, and catalysis applications.

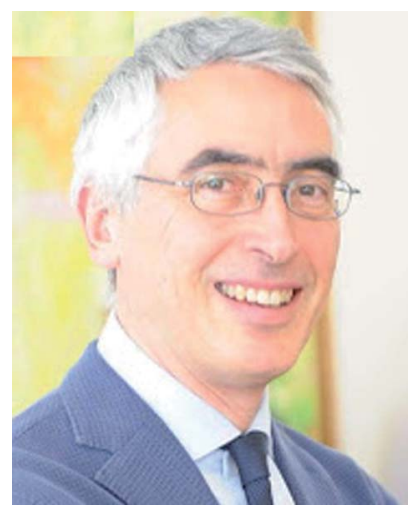

Stefano Superchi received his $\mathrm{PhD}$ in Chemistry from University of Pisa under the supervision of Professor Dario Pini and spent a research period at University of Waterloo (Canada) under the supervision of Professor Victor Snieckus. He then moved to University of Basilicata, where he is currently Associate Professor in Organic Chemistry. He is co-author of more than 90 publications including reviews and book chapters. His research is focused on absolute configuration assignment of chiral synthetic and natural products by chiroptical spectroscopy, on the development of chiral catalysts for asymmetric synthesis, and on the enantioselective synthesis of natural products and drugs. 


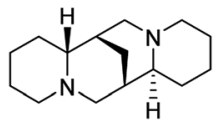

$(-)-L 1$<smiles>CN1C[C@@H]2C[C@H](C1)CN1CCCC[C@H]21</smiles>

L3

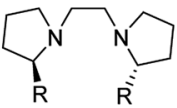

L6 R = Me L7 R $=\mathrm{CH}_{2} \mathrm{OMe}$

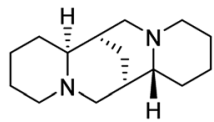

(+)-L1<smiles>CN(C)[C@@H]1CCCC[C@H]1N(C)C</smiles>

L4

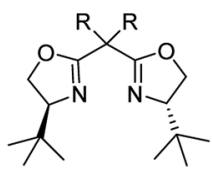

$\mathrm{L} 8 \mathrm{R}=\mathrm{Me}$

L9 $R=E t$

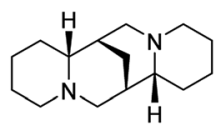

L2

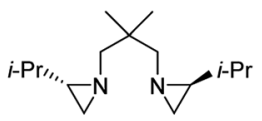

L5

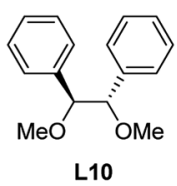

L10
Fig. 1 Chiral diamines employed in enantioselective carbolithiations.

diamines, e.g. $N, N, N^{\prime}, N^{\prime}$-tetramethylethylenediamine (TMEDA), which both enhance the reactivity of the organolithium reagents by reducing their aggregation and stabilize the carbolithiation product. ${ }^{13}$ Moreover, the use of diamines prompted the development of stereoselective versions of this reaction. In fact, in the presence of a chiral diamine ligand coordinating the organolithium intermediate, an asymmetric addition of the alkyllithium to the double bond occurs. The main drawback of the enantioselective carbolithiation is that generally, stoichiometric amounts of the chiral ligand are needed to achieve high enantioselection, thus posing some problems about the affordability and convenience of the reaction. In Fig. 1 the chiral ligands employed so far for asymmetric carbolithiation are reported (vide infra). Some of them, like (-)-sparteine (L1), its enantiomer $(+)-\mathbf{L 1}$, and its epimer $(-)$ - $\alpha$-iso-sparteine $(-)-\mathbf{L} 2$, are naturally occurring compounds, while the $(+)$-sparteine surrogate L3, diamines L4-L7, bis-oxazoline L8,L9 and diphenyldiether $\mathbf{L 1 0}$ are synthetic compounds. Among them, (-)-L1, readily available from the seeds of a variety of legumes, ${ }^{\mathbf{1 4 , 1 5}}$ has been the most powerful and widely used chiral ligand to control the enantioselectivity of organolithium reactions. ${ }^{\mathbf{1 6}}$

Aim of this review is to report a full overview on intermolecular carbolithiation processes, describing the most representative and synthetically valuable examples depending on the unsaturated substrates employed. The main focus is on the methodologies to achieve chemo-, regio- and stereo-control in the reaction, with a special attention on strategies to obtain asymmetric processes.

\section{Unfunctionalized alkenes}

\subsection{Cyclic trienes}

Cyclic trienes proved to be suitable substrates for carbolithiation reaction because in that case stabilization of the organolithium product by conjugation can occur, ${ }^{17}$ allowing to avoid the unwanted polymerization process. In fact, it was shown that

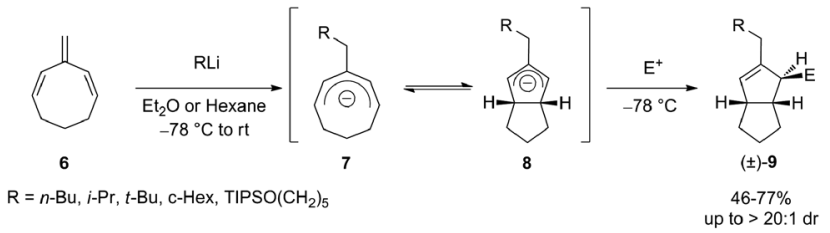

Scheme 4 Carbolithiation of cyclic triene 6

an intermolecular carbolithiation process of 1,3,5-cyclooctatriene (6) promotes a powerful cascade reaction sequence by which it is possible to synthesize functionalized cis-bicyclo [3.3.0]octenes. ${ }^{\mathbf{1 8 , 1 9}}$ From a mechanistic point of view, the addition of alkyllithium to the exocyclic double bond of the triene leads to the formation of a cyclooctadienyl anion 7 which undergo six-electron disrotatory ring closure, giving a bicyclic allyl anion 8. Subsequent electrophilic trapping of the latter provides cis-bicyclo[3.3.0]octenes $\mathbf{9}$ in diastereoselective manner (up to $20: 1$ d.r.) and in moderate to good yield (4677\%) (Scheme 4). This tandem electrocyclization/alkylation process allows formation of three new $\mathrm{C}-\mathrm{C}$ bonds and three stereocenters in a single pot. The reaction was found compatible with primary, secondary and tertiary alkyllithium reagents, and with several carbon and heteroatom electrophiles.

\subsection{Styrene derivatives}

Styrene (10a) represents the simplest aryl alkene useful as substrate for carbolithiation reactions. The reaction is highly dependent on the solvent. In fact, when reacting 10a with alkyllithium in THF a facile polymerisation occurs, even at $-78{ }^{\circ} \mathrm{C},{ }^{20}$ while the reaction is smoothly controllable in $\mathrm{Et}_{2} \mathrm{O}$, leading to the stabilised benzylic organolithium 11a and, after electrophile quenching, to the substituted product 12a in good yields. This shows that in $\mathrm{Et}_{2} \mathrm{O}$ the lithiated intermediate 11a is sufficiently stabilised to minimize polymerisation, whereas in THF it is not. Taylor and co-workers ${ }^{21,22}$ reported that styrene (10a) and several aryl-substituted styrene derivatives $\mathbf{1 0 b}-\mathbf{j}$ undergo efficient tandem carbolithiation-trapping reactions in $\mathrm{Et}_{2} \mathrm{O}$ at $-78{ }^{\circ} \mathrm{C}$ or at $-25^{\circ} \mathrm{C}$ (Scheme 5). In particular, $t$-BuLi and $s$-BuLi react with most of the aryl-substituted styrene derivatives at $-78{ }^{\circ} \mathrm{C}$, while primary alkyllithium reagents usually need higher temperatures $\left(\mathrm{ca} .-25{ }^{\circ} \mathrm{C}\right)$. Therefore, the reactivity of
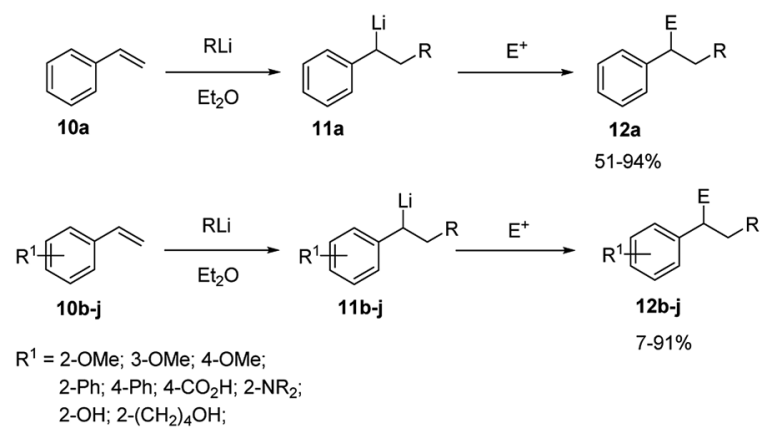

Scheme 5 Carbolithiation-trapping reaction of styrene (10a) and arylsubstituted styrenes $(10 \mathrm{~b}-\mathrm{j})$. 


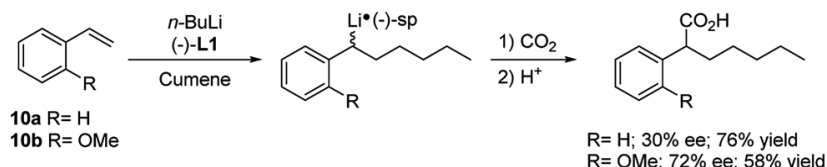

Scheme 6 Enantioselective carbolithiation-trapping reaction of styrene $10 \mathrm{a}$ and 0 -methoxy styrene $10 \mathrm{~b}$

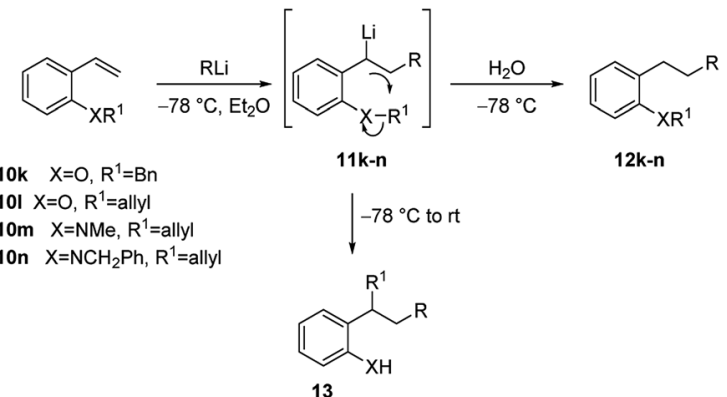

Scheme 7 Tandem carbolithiation/protonation or rearrangement of $10 \mathrm{k}-\mathrm{n}$.

different types of alkyllithium reagents was found to be: tertiary, secondary > primary $\gg$ alkenyl, methyl, phenyl. As expected, electron-donating groups at the ortho- or para-positions of the benzene ring, such as methoxy and dialkylamino moieties, deactivate the double bond towards alkyllithium addition, thus slowing down the reaction. However, in these cases, the reaction with $n$-BuLi can be facilitated by using TMEDA as cosolvent, which activate the alkyllithium for an efficient transformation.

The alkyllithium addition to styrene (10a) and its $o$ substituted derivatives $\mathbf{1 0 b}-\mathbf{j}$, followed by electrophilic trapping, has been investigated in the enantioselective version in the presence of (-)-L1 (Scheme 6). ${ }^{23} \mathrm{~A}$ low enantiomeric excess (up to $30 \%$ ) was observed with 10a at $-78{ }^{\circ} \mathrm{C}$ in cumene as solvent. Among the styrene derivatives, the best result ( $72 \%$ e.e.) was obtained using 2-methoxystyrene (10b) in cumene at $-95{ }^{\circ} \mathrm{C}$.

In both cases, 2 equivalents of the chiral diamine and $\mathrm{CO}_{2}$ as electrophile were used. Probably, the presence of an auxiliary binding site in 2-methoxy-substituted systems enables to stabilize the benzyllithium intermediate $\mathbf{1 1}$ by coordination, resulting in a greater stereoselectivity.

Furthermore, 2-benzyloxystyrene (10k) was shown to undergo efficient tandem carbolithiation/protonation with $t$ BuLi at $-78{ }^{\circ} \mathrm{C}$, affording $11 \mathbf{k}$, but when the reaction was allowed to warm to room temperature, before work-up, the carbolithiation reaction was followed by an intramolecular alkylation, leading to the corresponding 2-alkylphenol 12k (Scheme 7 and Table 1). The same process was found to occur with 2-allyloxystyrene (101), which undergoes efficient carbolithiation/intramolecular alkylation process with a range of alkyllithium reagents (Table 1). Phenyllithium, cyclohexyllithium and methyllithium, which do not add efficiently to 10a, can be used for this addition-alkyl transfer sequence in the presence of TMEDA. ${ }^{23,24}$ On the contrary, the 2-methoxy analogue 10b did not undergo methyl transfer on warming and the ortho-amino compounds (10m and 10n) did not transfer the $N$-methyl, $N$-benzyl or $N$-allyl groups.

This process also takes place with substituted allyloxy derivatives (Scheme 8). Thus, $Z$-butenol (Z-14) was converted into the rearranged products $Z$-15a-d with several alkyllithium reagents in diethyl ether, the allyloxy transfer occurring with retention of alkene configuration. In a similar manner the isomeric $E$-butenol $(E$-14) was converted into $E$-15a. In both reactions the double bond stereochemistry of the transferred group remains intact. ${ }^{22,24}$

The enantioselective carbolithiation of $\beta$-substituted nonfunctionalized styrenes was also investigated by Normant, Marek and co-workers (Scheme 9). ${ }^{25,26}$ Hence, the addition of various alkyl lithium to $(E)-\beta$-methyl styrene (16) in hexane and in the presence of $(-)-\mathbf{L} \mathbf{1}$ at $-\mathbf{1 5}{ }^{\circ} \mathrm{C}$, led, after hydrolysis, to the corresponding alkylated products $17 \mathbf{a}-\mathbf{c}$ in good yield and good enantioselectivity $(76-85 \%$ e.e. $)$ without polymerisation (Scheme 9a).

A slightly lower $70 \%$ e.e. was obtained for $17 \mathrm{a}$ when the reaction was performed in the presence of a catalytic amount of $(-)-\mathbf{L 1}(10 \mathrm{~mol} \%)$ at $0{ }^{\circ} \mathrm{C}$. The stereochemistry of the olefin is crucial for the enantioselectivity of the carbolithiation. Indeed, whereas the carbolithiation of the $(E)-\beta$-methyl styrene $(E-16)$ gave the $(S)$-alkylated product in $4 \mathrm{~h}$ at $-15{ }^{\circ} \mathrm{C}$, the same reaction on the $(Z)-\mathbf{1 6}$ led to the opposite enantiomer $(R)$ with a lower $28 \%$ e.e. Since the addition of alkyllithium on the (Z)isomer differs notably in rate from the one on the $(E)$-isomer,

Table 1 Tandem carbolithiation of $10 k-n$

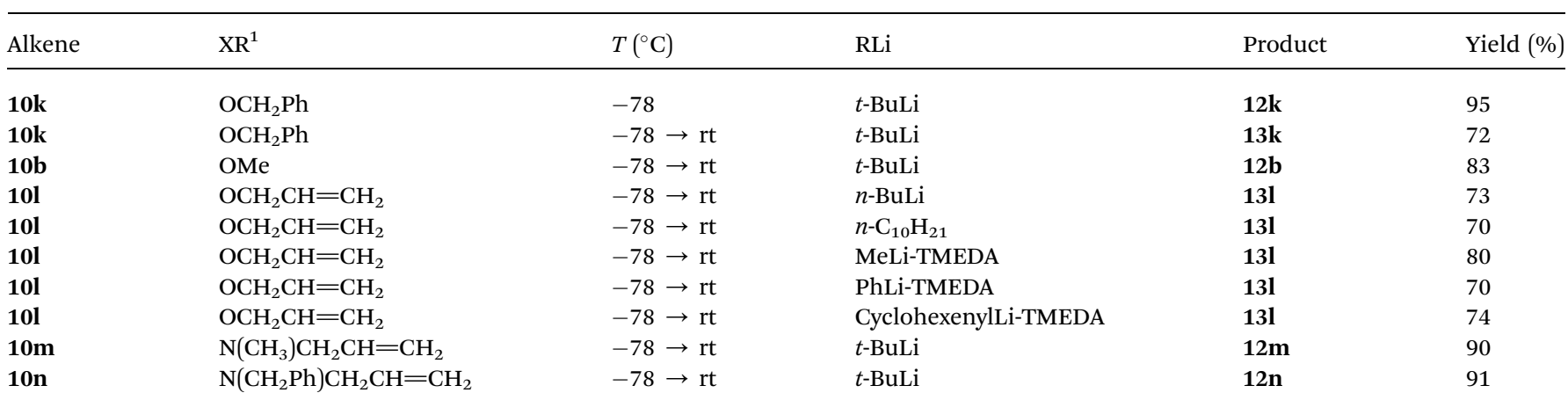



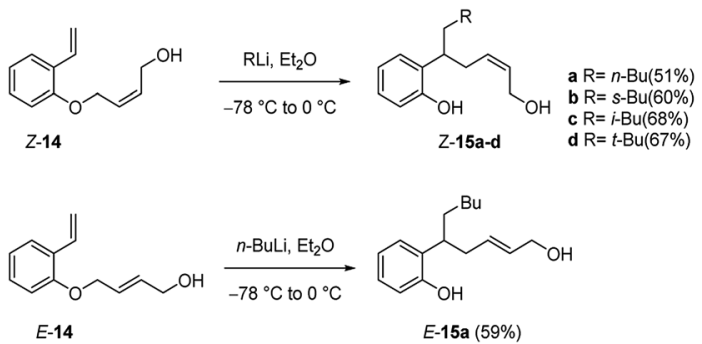

Scheme 8 Carbolithiation of $Z$ - and $E$-butenol 14

a)

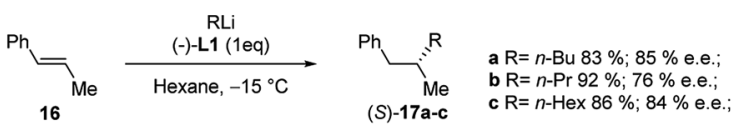

b)

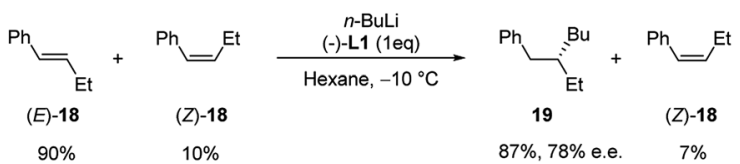

Scheme 9 Enantioselective carbolithiation of $\beta$-substituted nonfunctionalized styrenes (16 and 18).

a kinetic resolution of other $\beta$-alkylated styrene derivatives, such as $\beta$-ethyl styrene (18), was possible.

Therefore, carbolithiation with $n$-BuLi of 18 in a 90/10 E/Z ratio led, after hydrolysis, to the alkylated derivative 19 , derived from alkylation of $(E)-18$, in $87 \%$ yield and $78 \%$ e.e., while the $(Z)$-isomer was recovered intact (Scheme 9b).

Computational mechanistic studies on the asymmetric carbolithiation of $\beta$-methylstyrene (16) showed ${ }^{27}$ that in the nonfunctionalized system the high selectivity observed is due to repulsion between the (-)-L1 · alkyl lithium adduct and 16, upon their approximation in the diastereomeric transition states. In contrast, for the ortho-amino $\beta$-methylstyrene $(E)$-benzyl(2propenylphenyl)amine (38) (Scheme 15), X-ray analyses of intermediate lithium amides suggest that one side of the double bond is shielded by the ligand-coordinated amide group, leaving only one side available for approach of the chiral alkyl lithium adduct. Further investigation have shown that styrenes bearing unsaturated alkene or alkyne side chains at the $o$ position (20 or 22) undergo regioselective carbolithiation on the styrene unit, followed by 6-exo-trig (Scheme 10) or 6-exo-dig

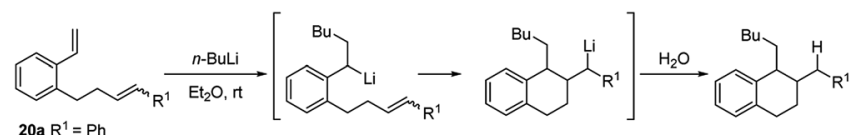
20a $R^{1}=P h$ $20 \mathrm{~b} \mathrm{R}^{1}=\mathrm{SiMe}_{3}$

21a $R^{1}=P h(71 \%$ from $E-20 a)$ 21b $\mathrm{R}^{1}=\mathrm{SiMe}_{3}(75 \%$ from $E-20 \mathrm{a})$ (72\% from Z-20a)

Scheme 10 Regioselective carbolithiation of styrenes bearing unsaturated alkene side chains at the o-position (20) followed by 6 -exo-trig cyclisation.

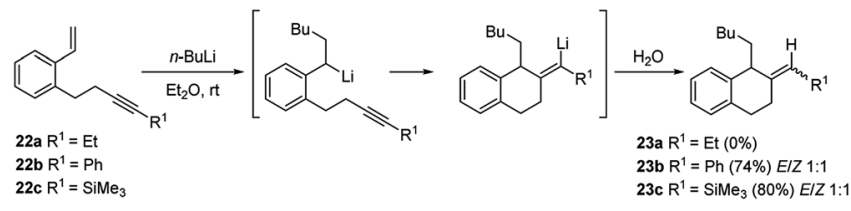

Scheme 11 Regioselective carbolithiation of styrenes bearing unsaturated alkyne side chains at the o-position (22) followed by 6 -exo-dig cyclisation.

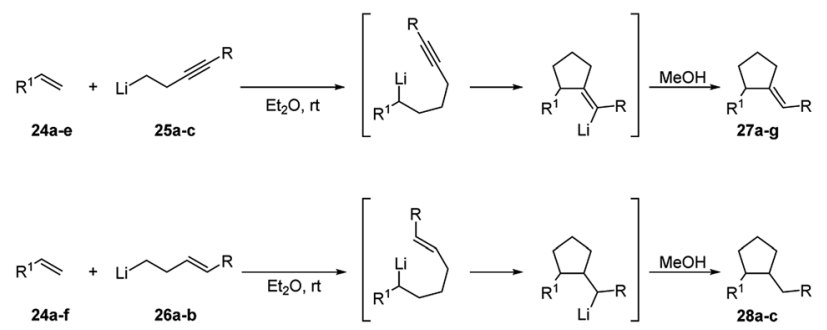

Scheme 12 Intermolecular carbolithiation-cyclisation process for cyclopentanes synthesis (see Table 2).

(Scheme 11) cyclisation to produce 1,2-disubstituted tetralins 21 or their unsaturated analogues, 23 respectively. ${ }^{28}$ The whole strategy represents an example of tandem intermolecular/ intramolecular carbolithiation process, which can also be stereoselective in the case of the corresponding vinylsilanes $(E)-20 b$ and $(Z)-\mathbf{2 0 b}$, where only the 1,2-trans-isomer of tetralin $\mathbf{2 1 b}$ was isolated regardless of which isomer of the silane was employed. Instead, the cyclisation did not occur using 22a as starting material, indicating that 6-exo-cyclisation is not facile with an inactivated alkyne.

The same tandem intermolecular/intramolecular carbolithiation protocol has been also extended to the synthesis of silacyclopentanes, prepared by the addition of alkyllithiums on vinyl(homoallyl)silanes or vinyl(homopropargyl)silanes. The initial intermolecular carbolithiation step is, then, followed by the intramolecular carbolithiation involving a 5-exo-trig or 5exo-dig cyclisation process. ${ }^{29}$ The same authors successfully explored even a tandem intermolecular/intramolecular carbolithiation sequence, starting from functionalized organolithium reagents and alkenes. Such a tandem process provides a general and versatile method for the preparation of complex cyclopentanes with a high level of functionality in a stereocontrolled manner. ${ }^{30}$ In order to facilitate the desired process, homopropargylic (25a-c) and homoallylic (26a-b) systems (Scheme 12) were chosen to avoid cyclisation of the initial organolithium reagents and "activated" alkenes $24\left(\mathrm{R}^{1}=\mathrm{Ar}, \mathrm{Ph}_{3} \mathrm{Si}, \mathrm{PhS}\right)$ were employed to ensure regioselectivity in the starting intermolecular carbolithiation. The addition of the functionalized organolithium to the double bond of the alkene led to the formation of a new organolithium intermediate, which after cyclisation and protonation afforded the desired cyclopentanes 27 and 28 in moderate to high yields (45-82\%) and often with excellent stereocontrol (Scheme 12 and Table 2). The prevalence of the $E$ alkene in the products $\mathbf{2 7}$ is consistent with the accepted syn- 
Table 2 Intermolecular carbolithiation-cyclisation process for synthesis of cyclopentanes 27 or 28

\begin{tabular}{|c|c|c|c|c|c|}
\hline \multicolumn{2}{|l|}{ Alkene } & \multicolumn{2}{|c|}{ Organolithium } & Product & Yield (\%) $(E / Z)^{a}$ or $(\text { trans } / c i s)^{b}$ \\
\hline 24a & $\mathrm{Ph}$ & $25 b$ & $\mathrm{Ph}$ & $27 \mathbf{b}$ & $53(70: 30)$ \\
\hline $24 a$ & $\mathrm{Ph}$ & $25 c$ & $\mathrm{SiMe}_{3}$ & $27 \mathrm{c}$ & $82(76: 24)$ \\
\hline $24 b$ & $2-\mathrm{Np}$ & $25 b$ & $\mathrm{Ph}$ & $27 d$ & $62(56: 44)$ \\
\hline 24e & $\mathrm{C}_{6} \mathrm{H}_{4}\left(o-\mathrm{CH}_{2} \mathrm{NMe}_{2}\right)$ & $25 c$ & $\mathrm{SiMe}_{3}$ & $27 \mathrm{~g}$ & $56(85: 15)$ \\
\hline 24a & $\mathrm{Ph}$ & 26d & $\mathrm{Ph}$ & $28 a$ & $52(50: 50)$ \\
\hline 24a & $\mathrm{Ph}$ & $(E)-26 \mathbf{e}$ & $\mathrm{SiMe}_{3}$ & $28 b$ & $52(100: 0)$ \\
\hline $24 a$ & $\mathrm{Ph}$ & $(Z)-26 \mathrm{e}$ & $\mathrm{SiMe}_{3}$ & $28 b$ & $45(100: 0)$ \\
\hline $24 f$ & $\mathrm{SiPh}_{3}$ & $26 d$ & $\mathrm{Ph}$ & $28 \mathrm{c}$ & $45(100: 0)$ \\
\hline
\end{tabular}

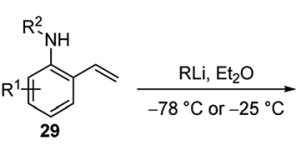

$\mathrm{RLi}=n-\mathrm{BuLi}, s-\mathrm{BuLi}, t-\mathrm{BuLi} ;$ $\mathrm{R}^{1}=\mathrm{H}, \mathrm{F}, \mathrm{OMe}, \mathrm{Me}$ $\mathrm{R}^{2}=\mathrm{Boc}, \mathrm{Et}, \mathrm{Bn}$.
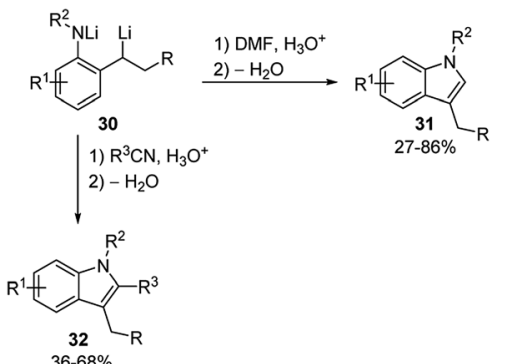

Scheme 13 Carbolithiation-trapping reactions of 0 -aminostyrenes (29).

carbolithiation mechanism. However, when a diethyl ether/THF (3/1) solvent system was employed the corresponding $Z$-isomer of 27a was obtained as the major product. To explain such result, it was hypothesized that THF solvation increases the effective size of the lithium substituent eventually leading to vinyl inversion. The fully stereoselective formation of $27 \mathrm{e}$ and 27f is determined by intramolecular coordination of the intermediate vinyllithium to oxygen and sulfur, respectively. The lack of stereoselectivity observed in the reaction of styrene (10a) and 26a is probably due to a $\pi$-stacking effect, which provides a further stabilization for cis-28a. The intermolecular

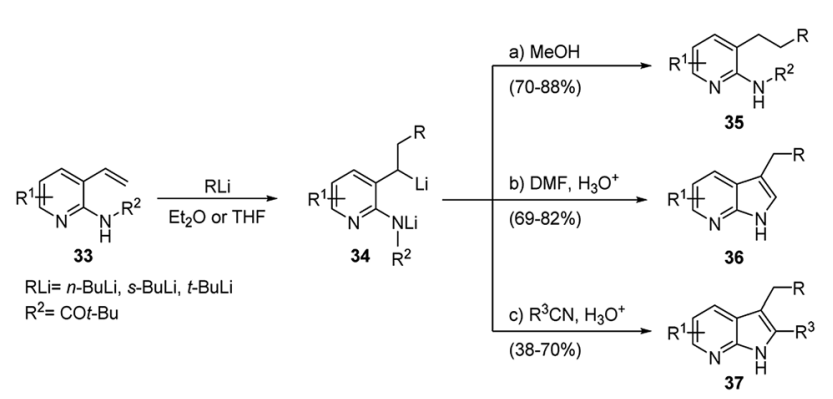

Scheme 14 Carbolithiation/protonation reaction of 3-vinylpyridin-2ylamines (33). carbolithiation reactions of the $o$-aminostyrenes 29 resulted to be efficient and, interestingly, allowed the preparation of functionalized indole ring systems (31 and 32) by means of a one-pot process. ${ }^{31,32}$

This methodology involves a cascade reaction sequence constituted by an initial $\mathrm{NH}$ deprotonation, followed by an alkyllithium addition to the styrene double bond and a subsequent electrophile trapping of the organolithium intermediate generating a carbonyl moiety. A subsequent in situ ring closure and dehydration provide the indole ring (Scheme 13). This is an efficient and versatile method allowing to introduce diverse substituents at all positions of the indole scaffold. Indeed, the procedure was shown to be successful with a wide range of both $\mathrm{C}$ and $\mathrm{N}$ substituents on the $o$-aminostyrenes. Moreover, it was tolerant to the reactivity range of alkyllithiums such as $t$-, $s$-, and $n$-BuLi. The electrophiles used were DMF, which generated indole products 31 unsubstituted at C-2, and nitriles, which allowed to incorporate the nitrile substituent at $\mathrm{C}-2$ position of the indole 32 (Scheme 13).

The same procedure was extended to the synthesis of 7azaindoles starting from 3-vinylpyridin-2-ylamines 33, which undergo efficient carbolithiation/protonation reaction (Scheme 14). As mentioned above, the methodology involves a reaction sequence consisting of a controlled carbolithiation of the vinyl double bond, subsequent trapping of the formal di-anion

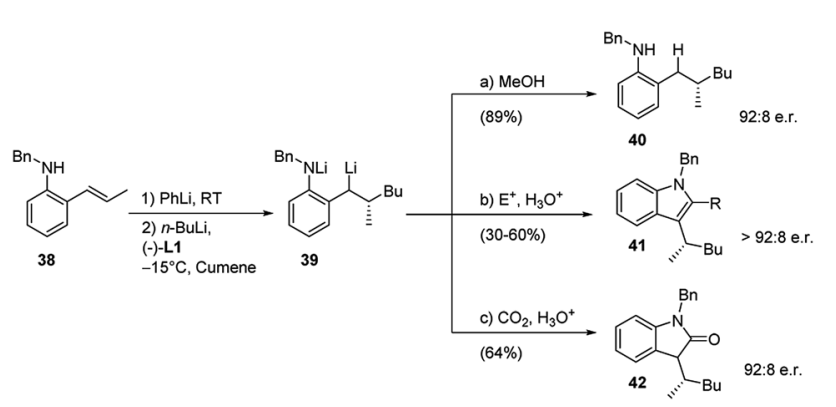

Scheme 15 Enantioselective carbolithiation of $(E)$-2-propenylarylamine 38 . 
Table 3 Enantioselective carbolithiation and electrophile trapping of 38

\begin{tabular}{llllll}
\hline Substrate & $\mathrm{E}^{+}$ & $\mathrm{R}$ & Product & Yield (\%) & e.r. \\
\hline $\mathbf{3 8}$ & $\mathrm{DMF}$ & $\mathrm{H}$ & $\mathbf{4 1 a}$ & 60 & $92: 8$ \\
$\mathbf{3 8}$ & $\mathrm{MeCON}(\mathrm{OMe}) \mathrm{Me}$ & $\mathrm{CH}_{3}$ & $\mathbf{4 1 b}$ & 30 & $93: 7$ \\
$\mathbf{3 8}$ & $\mathrm{PhCN}$ & $\mathrm{Ph}_{3}$ & $\mathbf{4 1 c}$ & 38 & $93: 7$ \\
$\mathbf{3 8}$ & $\mathrm{MeC}(\mathrm{OEt})_{2} \mathrm{CN}$ & $\mathrm{COCH}_{3}$ & $\mathbf{4 1 d}$ & 51 & $92: 8$ \\
$\mathbf{3 8}$ & $\mathrm{C}_{4} \mathrm{H}_{6} \mathrm{O}_{2}$ & $\left(\mathrm{CH}_{2}\right)_{3} \mathrm{OH}$ & $\mathbf{4 1 e}$ & 45 & $92: 8$ \\
$\mathbf{3 8}$ & $t$ - $\mathrm{BuCN}$ & $t-\mathrm{Bu}$ & $\mathbf{4 1 f}$ & 50 & $91: 9$
\end{tabular}

intermediate with a suitable electrophile, again DMF and substituted nitriles, followed by an in situ ring closure and dehydration (Scheme 14). ${ }^{33}$

The reaction sequence allows aryl, heteroaryl, alkyl and keto substituents to be included at different positions around the heterocycle. Also, this reaction sequence proved to be tolerant for different alkyllithiums $(t-, s$-, or $n$-butyl), leading to the preparation of 3-substituted 7-azaindoles 36 in good to excellent yields (69-82\%) when DMF was used as electrophile (pathway b), and to 2,3-disubstituted 7-azaindoles 37 in moderate to good yields (38-70\%) using nitriles (pathway c).

The procedure above was also applied to $o$-substituted $\beta$ methylstyrenes $\mathbf{3 8}$ even in an enantioselective version. The enantioselective carbolithiation of the $(E)$-2-propenylarylamine (38), mediated by (-)-L1, allowed to generate chiral lithiated intermediate 39 which have wide synthetic potential (Scheme 15). ${ }^{34}$ Indeed, intermediate 39 can undergo a series of further in situ transformations, reacting with electrophiles to afford a collection of products sharing a common stereogenic centre.

The stereogenic centre, formed in high enantiomeric ratio in the first carbolithiation step, is carried through the cascade reaction sequence to the final products $(\mathbf{4 0 , 4 1}$ and 42$)$ and it is independent of electrophile used. This methodology provided access to the synthesis of structurally diverse chiral anilines (40), indoles (41), and indolones (42) all with an e.r. of $92: 8$. The heterocyclic syntheses involve an enantioselective alkene carbolithiation and subsequent trapping of the intermediate organolithium with a suitable electrophile, followed by an in situ ring closure and dehydration to generate the indole or indolone rings. Moreover, a study of the amount of $(-)-\mathbf{L 1}$ additive employed revealed its crucial role in gaining selectivity. The best results were obtained when an equal ratio of organolithium (PhLi $+n$-BuLi) to (-)-L1 was used. ${ }^{35}$ After the carbolithiation step, the organolithium intermediate 39 was reacted with $\mathrm{MeOH}$ or other several electrophiles, leading to the chiral aniline $\mathbf{4 0}$ or indole 41, respectively. If $\mathrm{CO}_{2}$ is used as electrophile it is also possible to obtain the chiral indolone $\mathbf{4 2}$.

The exploited electrophiles and the corresponding results are summarized in the Table 3 .

The same protocol allowed an easy access to four further important heterocyclic classes, namely, the isoquinolines 46 and isoquinolinones 47 (Scheme 16), benzofurans 51 (Scheme 17), and isobenzofuranones 56 (Scheme 18), starting from the corresponding prochiral $\beta$-methylstyrene substrates containing

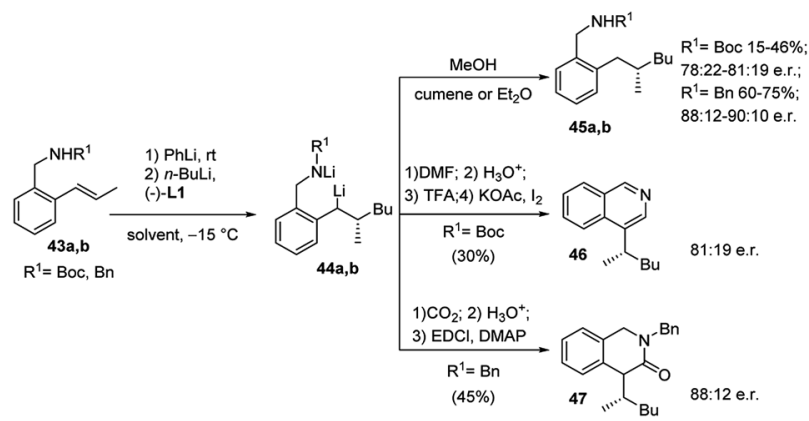

Scheme 16 Synthesis of isoquinolines 46 and isoquinolinones 47 by using carbolithiation-trapping-cyclisation sequence.

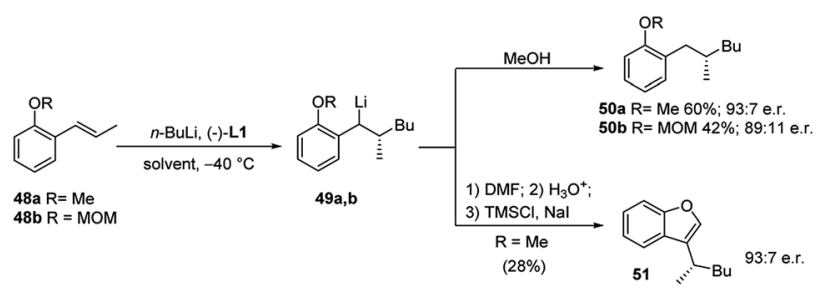

Scheme 17 Synthesis of benzofurans 51 by using carbolithiationtrapping-cyclisation sequence.

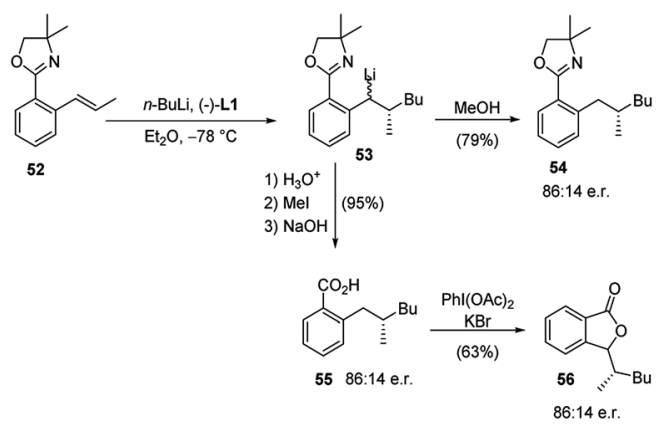

Scheme 18 Synthesis of isobenzofuranones 56 by using carbolithiation-trapping-cyclisation sequence.

$o$-aminomethyl 43, ether 48 and oxazoline 52 substituents, respectively. As the chiral centre, generated during the $\mathrm{C}-\mathrm{C}$ bond formation in the first carbolithiation step, is carried through the electrophile reaction sequence to the final products, the enantioselectivity is solely dependent upon the alkyllithium addition. ${ }^{36}$ Moreover, this approach was shown to be tolerant of several alkyllithium and both electron donating and withdrawing substituents on the aryl ring. ${ }^{34,35}$ It is noteworthy that the $o$-methoxy-substituted $\beta$-methylstyrene (48a) gave a better selectivity (94: 6 e.r.) and yield (60\%) than the MOMsubstituted $\mathbf{4 8 b}$, which would be considered a stronger coordinating group (Scheme 17). ${ }^{36}$

The approach reported by O'Shea et al. then provides a method to synthesize chiral aromatic and benzoheterocyclic compounds. The value of such a method lies in the wideranging possibilities for the use of the chiral lithiated 

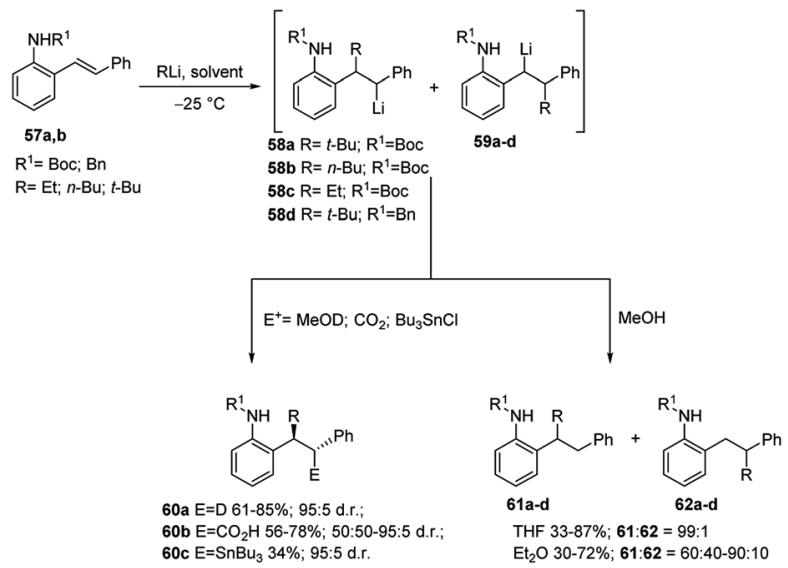

Scheme 19 Carbolithiation of $O$-amino substituted $(E)$-stilbenes $57 a, b$.

intermediates, formed by means of an enantioselective carbolithiation of $o$-substituted $\beta$-methylstyrenes.

As previously shown, carbolithiation of styrenes and $\beta$ alkylstyrenes is regiospecific with the more stabilised benzylic lithiated regioisomer generated exclusively in each case. In contrast, by addition of alkyllithium to unsymmetrical stilbenes two different benzyllithium intermediates could be formed. However, in the case of $o$-amino substituted $(E)$-stilbenes $\mathbf{5 7}$ the carbolithiation regioselectivity can be tuned by the solvent. In fact, in THF the single regioisomer $\mathbf{5 8}$, lithiated at the carbon $\alpha$ to the phenyl ring is obtained. Instead, the use of other solvents, such as diethyl ether, led to a pronounced loss in regioselectivity, giving rise to a mixtures of both possible regioisomers (Scheme 19). ${ }^{37,38}$

The solvent choice allows also to control the diastereoselectivity of carbolithiation/electrophile substitution sequence (Scheme 19). ${ }^{38}$ Indeed, the carbolithiation in THF of unsymmetrical 1,2-disubstituted alkenes, such as stilbenes $\mathbf{5 7}$, generated the organolithium species $\mathbf{5 8}$ with two contiguous stereocentres, which can be trapped with several electrophiles, leading to two possible diastereomers. High levels of diastereoselectivity have been obtained in THF using different electrophiles such as $\mathrm{MeOD}, \mathrm{CO}_{2}$, and $\mathrm{Bu}_{3} \mathrm{SnCl}$, with the major diastereomer $\mathbf{6 0}$ being the one in which the electrophile and alkyl group are anti to each other. It was also shown that the diastereoselectivity is influenced by both the ortho-amino substituent and the employed alkyllithium, with $N$-Boc substituent and $t$-BuLi giving the best results in terms of diastereoselectivity. Probably, this is due to the higher ability of the Boc group to give intramolecular chelation with the benzylic lithium. However, when the organolithium species $\mathbf{5 8 b}$ was quenched with $\mathrm{CO}_{2}$, no diastereoselectivity was observed and the substitution product 60b was isolated as an equal mixture of diastereoisomers (Scheme 19).

Furthermore, the regioselective carbolithiation of substituted $o$-amino- $(E)$-stilbenes $\mathbf{5 7}$ was exploited in a cascade methodology involving a regiospecific alkyllithium addition to the alkene, subsequent trapping of the organolithium intermediate with a suitable electrophile, followed by an in situ ring closure and dehydration to provide the quinoline scaffold (Scheme 20). ${ }^{37,38}$ By using this method it was possible to synthesize substituted 3,4-dihydro-quinolin-2-ones 63, 1,2,3,4tetrahydroquinolines 64, di- or tri-substituted quinolines 65 (Scheme 20) and di- or tri-substituted $N$-benzyl-1,4dihydroquinolines 66 (Scheme 21). It was found that the treatment of the acyclic carboxylic acids $60 \mathrm{~b}$ with aqueous $12 \mathrm{M} \mathrm{HCl}$ led to removal of the Boc group and intramolecular cyclisation, allowing isolation of 3,4-dihydro- $1 H$-quinolin-2-ones $\mathbf{6 3}$ (Scheme 20, route a). The same product $\mathbf{6 3}$ can be obtained by raising the reaction temperature to either $0{ }^{\circ} \mathrm{C}$ or $\mathrm{RT}$, after the carbolithiation step at $-25^{\circ} \mathrm{C}$ (Scheme 20 , route b). Indeed, the increase in temperature gives rise to cyclisation, resulting by intramolecular acyl nucleophilic substitution of the benzylic lithium centre at the $t$-Boc group. In both cases, the predominant isomer was the 3,4-trans one. The reaction of lithiated intermediates with DMF followed by treatment with aqueous acid solutions provided a versatile synthesis of the 1,2,3,4-

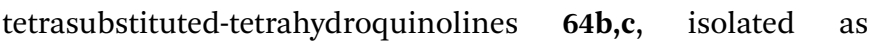
mixture of the two diastereomeric products, 2,3-cis-3,4-trans and 2,3-trans-3,4-trans (Scheme 20, route c). Dehydration and in situ aromatisation of $\mathbf{6 4 b}, \mathbf{c},(\mathrm{R}=n$-Bu or Et $)$, by treatment with aqueous $\mathrm{HCl}$ allowed the synthesis of 3,4-disubstituted quinoline rings 65b,c. Surprisingly, the $t$-Bu substituted analogue 64a,c $(\mathrm{R}=t$-Bu) yielded only the mono-substituted 3-phenylquinoline 65a $\left(\mathrm{R}^{1}=\mathrm{H}\right)$, indicating that the aromatic ring was generated by loss of the $t$-Bu group. The same result was also obtained when milder acidification conditions were employed (5 $\mathrm{M} \mathrm{HCl}$ in THF). Compounds 65 can also be prepared directly from lithiated intermediates $\mathbf{5 8 a - c}$ without isolation of intermediate 64, by using DMF or nitriles as electrophiles, thus providing the opportunity to introduce different substituents in 2-position on the quinoline ring (Scheme 20, route d). Moreover, the reaction of $N$-benzyl substituted lithiated intermediates 59d-f with DMF or nitriles as electrophiles provided a direct entry into the 1,4-dihydroquinoline class 66 (Scheme 21). ${ }^{37}$

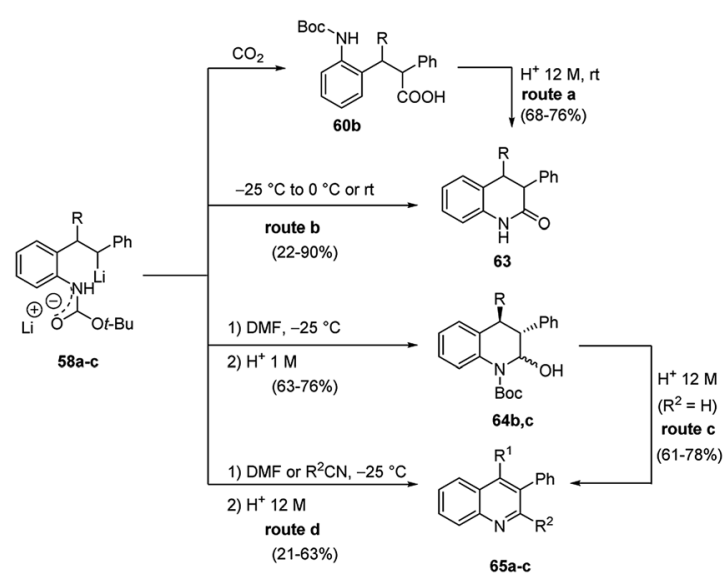

Scheme 20 Trapping of the organolithium intermediates $58 a-c$ with a suitable electrophile, followed by an in situ ring closure and dehydration to provide the quinoline and tetrahydroquinoline scaffold. 


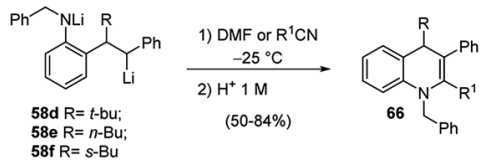

Scheme 21 Reaction of $N$-benzyl substituted lithiated intermediates $58 \mathrm{~d}-\mathrm{f}$ with DMF or nitriles.

\section{Functionalized alkenes}

\subsection{Cinnamyl derivatives}

Carbolithiation reaction of cinnamyl alcohol (67) (Scheme 22) was reported for the first time in 1990 by Kuwajima and coworkers. ${ }^{39}$ The addition of $n$-BuLi was followed by electrophilic substitution using several electrophiles. The whole tandem reaction was found to proceed in highly diastereoselective manner, with the preferential formation of the $s y n$-isomer in every investigated case. Surprisingly both (E)-67 and (Z)-67 olefin substrates generate the same syn-product, showing that the selectivity is not affected by the stereochemistry of the starting alkene. In order to rationalize the syn-selectivity the authors supposed the formation of two five-membered cyclic benzyllithium intermediate species having a $\mathrm{sp}^{2}$-like carbon to which two lithium atoms coordinate from both upper and lower side. Among them, the trans dilithium intermediate 68 seems to be energetically favored and the electrophile selectively substitutes the lithium atom from the upper site to afford 69. The stereochemical outcome of the carbolithiation-trapping with electrophiles of cinnamylamines 70 (Scheme 23) was later described by Normant, Marek and co-workers. ${ }^{40}$ Also in this case, the high diastereoselectivity observed in the reaction can be ascribed to the thermodynamic control of the organolithium intermediate $\mathbf{7 1}$, since the same anti-product starting from both $(E)-70$ and $(Z)-70$ cinnamylamine was obtained. According to the authors, this can be explained considering that, after thermodynamic equilibration, the tertiary amino group $\left(\mathrm{NEt}_{2}\right.$ or $\left.\mathrm{NMe}_{2}\right)$ on the $\gamma$ position blocks the configuration of the benzyllithium through coordination. This allows a diastereoselective introduction of electrophiles with retention of configuration. Moreover, the diastereomeric ratio is dependent on the reaction temperature in hexane, raising from $80: 20$ to $95: 5$ with increasing temperature from $-30{ }^{\circ} \mathrm{C}$ to $\mathrm{rt}$.

Summarizing, cinnamyl amines gave anti-products while the alkoxide compounds were shown to yield mainly the synsubstituted diastereomers. This result was attributed by the authors to the oligomeric nature of the carbolithiation products of cinnamyl alcohol 68, which exist as dimer and higher aggregates under the reaction conditions. ${ }^{\mathbf{4 1 , 4 2}}$

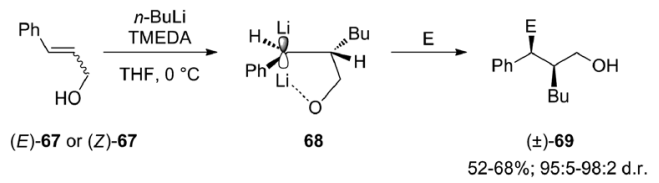

Scheme 22 Diastereoselective carbolithiation-trapping reaction of cinnamyl alcohol (67).
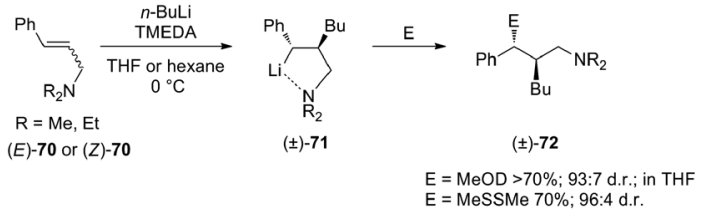

Scheme 23 Diastereoselective carbolithiation-trapping reaction of cinnamylamines 70 .

Normant, Marek and co-workers also described the asymmetric carbolithiation of cinnamyl alcohol 67 or amines 70 promoted by stoichiometric amounts of the chiral ligand (-)-L1 and using primary and secondary alkyllithium (Scheme 24a). ${ }^{3,26,43}$ Contrary to what was described above, the authors reported that in this case the benzylic organolithium 68 or 71, obtained from alcohol 67 and amines 70, respectively, undergoes electrophilic substitution with inversion of configuration, leading to $s y n$-isomers of the products 69 or 72 with up to $85 \%$ e.e. and a high (>96\%) diastereomeric purity. Therefore, such a simple one-pot process allows to introduce two contiguous stereogenic centres on an acyclic system with a good level of stereoselectivity. The best results were obtained using cumene as solvent, since $\mathbf{L} 1$ shows the most pronounced effect in the absence of coordinating solvents, such as ether or THF, and using primary alkyl lithium ( $n$-BuLi), being the RLi-L1 interaction stronger with sterically less demanding organolithium derivatives. Moreover, the stereochemical outcome of the reaction was found to be closely dependent on the stereochemistry of the starting olefin. Indeed, whereas the asymmetric carbolithiation of cinnamyl alcohol $((E)-67)$ gave the $(S)$ alkylated product 69 a with $83 \%$ e.e. (Scheme $24 \mathrm{~b}$ ), the reaction of the (Z)-67 isomer led mainly to $(R)$-enantiomer of $69 \mathbf{a}$ (Scheme 24c). Interestingly, when the last reaction was performed using catalytic amounts (5\%) of (-)-L1 products with comparable e.e. were obtained. Furthermore, the high stereospecificity of this process allows obtaining both enantiomers of the product by means of the same enantiomer of the ligand. Subsequently, the enantioselective carbolithiation of cinnamyl alcohol reported in Scheme 24 was also reinvestigated by using (+)-sparteine surrogate L3. Therefore, the addition of the complex $n$-butyllithium/L3 to cinnamyl alcohol $((E)-67)$ in cumene at $0{ }^{\circ} \mathrm{C}$ gave alcohol $(R)-69$ a in $71 \%$ yield with $74 \%$ e.e., that is with essentially opposite stereoinduction in respect to that obtained by Normant with (-)-L1 (82\% yield, $83 \%$ e.e. in favour of $(S)$-69a). ${ }^{44}$

In a syn-addition, typical for carbometallation, to the $(E)$ cinnamyl alcohol (67), the chiral complex $n$-butyllithium/(-)-L1 attacks the $\mathrm{C}=\mathrm{C}$ double bond from the Si-face (Scheme 25) and the resulting five-membered ring chelate complex bears phenyl and butyl residues in a cis-orientation. Hence, the configurationally labile benzyllithium derivative epimerizes, leading to the formation of the more stable trans-substituted ring chelate product. The subsequent protonation produced the $(S)$-configured alcohol with $80 \%$ e.e., whereas methylation, which occurs with inversion of configuration, affords the disubstituted $(S, S)$ alcohol with $82 \%$ e.e. Thus the enantiofacial differentiation 
a)
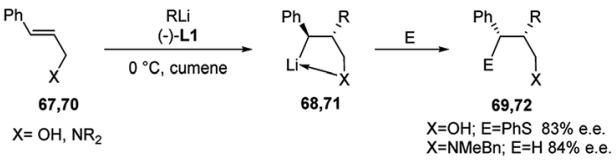

b)
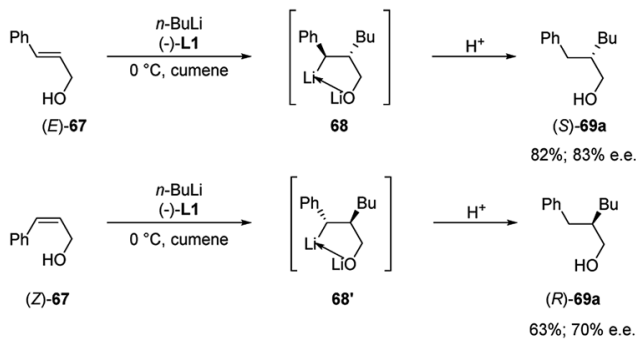

Scheme 24 Asymmetric carbolithiation of cinnamyl alcohol or amines (67 or 70) (route a). Carbolithiation of (E)-67 (route b) and (Z)-67 (route c)

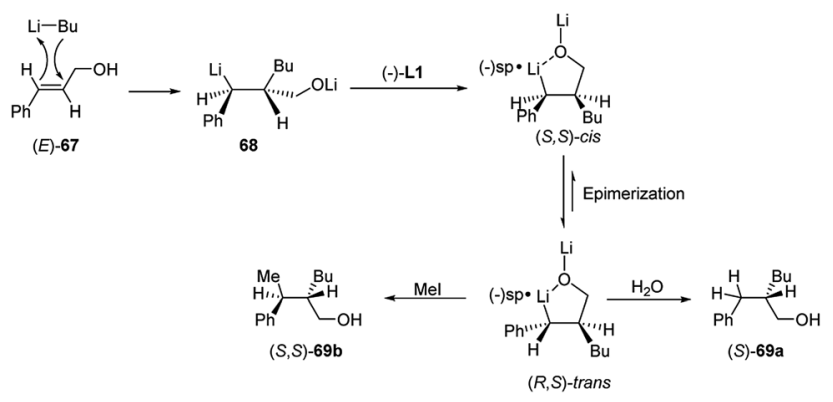

Scheme 25 Enantioselective carbolithiation of $(E)-67$.

promoted by (-)-L1 ligand in the addition to a double bond in a conformationally restricted complex, determines the stereochemical outcome of the reaction, since a configurationally stable stereocenter is created at C-2. Consequently, the $(Z)$ alcoholate leads to the opposite series of enantiomers. ${ }^{\mathbf{1 5}}$

Highly enantioselective carbolithiation of the dimethyl acetal of the $(E)$-cinnamyl alcohol 73 , mediated by either stoichiometric or catalytic (1.0 or $10 \mathrm{~mol} \%$ ) amount of (-)-L1, was also reported (Scheme 26). ${ }^{45}$ The acetal group gives the advantage to enforce the proximity effect and consequently to increase the e.e. Through this strategy it is possible to obtain, after hydrolysis at low temperature, the corresponding alkylated alcohols 75 in $90-95 \%$ e.e. and in $50-80 \%$ yields using stoichiometric amounts of the chiral ligand and comparable $92 \%$ e.e. in the presence of $10 \mathrm{~mol} \%$ of $(-)-\mathbf{L 1}$, whereas a slight loss of enantioselectivity ( $85 \%$ e.e.) was observed with only $1.0 \mathrm{~mol} \%$ of chiral ligand.

The use of the acetal allows the reaction to proceed at $-50{ }^{\circ} \mathrm{C}$ instead of $0{ }^{\circ} \mathrm{C}$, as for the carbolithiation of the corresponding alcohol 67. Furthermore, simply by warming the reaction mixture to rt, the benzylic organolithium intermediate, formed by the carbolithiation step, undergoes a 1,3-elimination, affording the chiral disubstituted cyclopropane 76 in 90-93\% e.e. and in 59-66\% yields (Scheme 26). In summary, when the chelating moiety is a dimethyl-methoxymethyl ether, the formed benzylic organolithium species is thermally labile at rt,

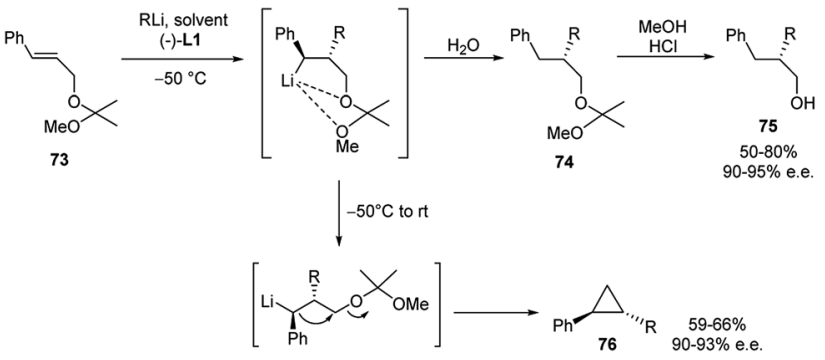

Scheme 26 Enantioselective carbolithiation of the dimethyl acetal of the $(E)$-cinnamyl alcohol 73 .

leading to a chiral disubstituted cyclopropane, via an internal nucleophilic substitution. The alkyl and phenyl groups were found to be anti to each other. That is because in this transformation, the initially formed stereogenic centre $\mathrm{C}-\mathrm{R}$ is invariant, whereas the benzylic carbon is free to epimerize and to promote the formation of the thermodynamically more stable trans cyclopropane..$^{3,43,45}$

In a similar way, Marek and co-workers developed the first catalytic asymmetric and stereoselective synthesis of chiral $(E)$ trans disubstituted-vinylcyclopropane, via enantioselective carbolithiation of dienyl systems 77 followed by a 1,3-intramolecular elimination. ${ }^{46}$

The addition of a $n$-alkyllithium ( $n$-BuLi or $n$-HexLi) in hexane in the presence of $10 \mathrm{~mol} \%$ of $(-)-\mathbf{L 1}$ at $-10{ }^{\circ} \mathrm{C}$ led to the formation of the allylic organolithium derivatives $\mathbf{7 8}$ as a mixture of cis- and trans-diastereomers. After the carbolithiation step, a warming of the reaction mixture to rt enabled to obtain the vinylcyclopropanes $\mathbf{7 9}$ both in moderate to good e.e.'s (50-83\%) and yields (45-70\%) (Scheme 27). The reaction was shown to be highly stereoselective because only the $(E)$ configured double bond was obtained and, furthermore, the alkyl and vinyl groups in the cyclopropane derivatives were trans to each other. The authors assumed that the absolute configuration of the initially formed stereogenic $\mathrm{C}-\mathrm{R}$ centre is due to the enantioselective carbolithiation step and unchanged in the 1,3-elimination reaction. On the contrary, the allylic lithium is free to epimerize through a dynamic thermodynamic diastereomer resolution, promoting the formation of the thermodynamically more stable $(E)$ - and trans-disubstituted vinylcyclopropanes 79. The whole process involves both the enantiofacial choice of a dienyl system by the chiral

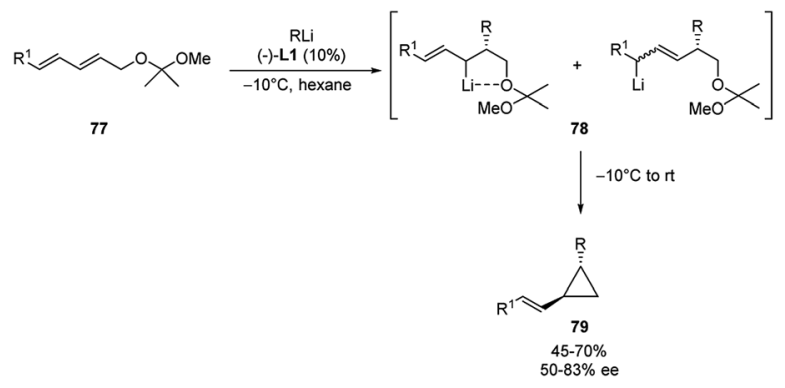

Scheme 27 Asymmetric carbolithiation of 77 and synthesis of 79 


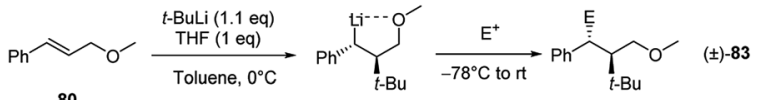

$$
\begin{aligned}
& E=H 79 \% ; E=D \quad 73 \% \text {; antilsyn 90:10 } \\
& \mathrm{E}=\mathrm{COOH} 79 \% \text {; antilsyn } 85: 15 \\
& \mathrm{E}=\mathrm{SCH}_{3} 61 \% \text {; anti/syn 98:2 } \\
& \mathrm{E}=\mathrm{CH}_{3} 60 \% \text {; anti/syn 13:87 } \\
& \mathrm{E}=n-\mathrm{Bu} 49 \% ; \text { antilsyn 2:98 }
\end{aligned}
$$

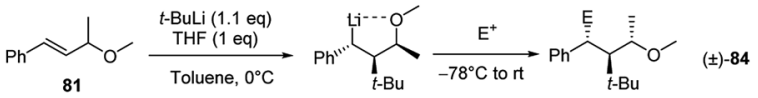

$$
\begin{aligned}
& E=H \text { 84\%; } E=D \text { 83\%; anti/syn 94:6 } \\
& \mathrm{E}=\mathrm{COOH} 75 \% \text {; antilsyn } 73: 27
\end{aligned}
$$

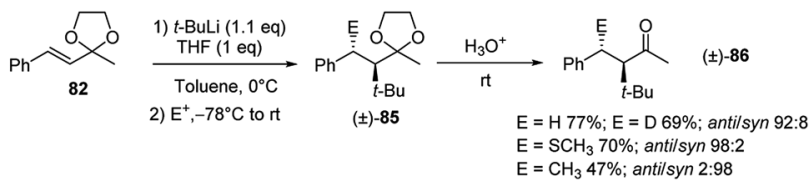

Scheme 28 Carbolithiation and subsequent electrophilic trapping of 80,81 and 82 .

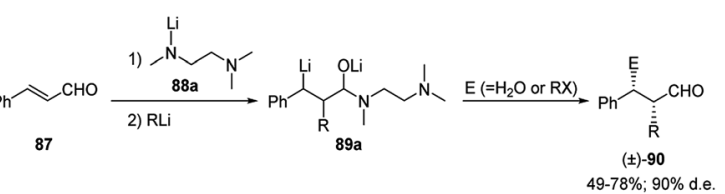

Scheme 29 Carbolithiation-trapping reaction of cynnamaldehyde (86).

organolithium and the stereoselective 1,3-elimination into the corresponding cyclopropane.

The diastereoselectivity in the carbolithiation reaction of cinnamyl methyl ethers was also investigated. ${ }^{41}$ As shown in Scheme 28, the addition of $t$-BuLi to cinnamyl methyl ether $(\mathbf{8 0})$, $\alpha$-methylcinnamyl methyl ether (81) and 2-cinnamyl-2-methyl1,3-dioxolane (82) followed by electrophilic substitution smoothly occurs. The whole tandem process proceeds with high diastereoselectivity, providing the anti-products (relative configuration between $t$ - $\mathrm{Bu}$ and $E$ ) when $\mathrm{MeOD}, \mathrm{CO}_{2}$ and MeSSMe were used as electrophiles. Instead, the use of alkyl halides led to the syn-isomers, suggesting that in these cases the subsequent electrophilic substitution reaction occurs with inversion of configuration at the lithiated centre. Acid hydrolysis of the acetals 85, obtained from carbolithiation/trapping with electrophiles of $\mathbf{8 2}$, releases the ketones in acceptable yields and in highly diastereoselective manner.

Among the cinnamyl derivatives, cinnamaldehyde (87) was also shown to be a suitable substrate for the carbolithiation reaction. Indeed $\mathbf{8 7}$, protected as a lithium aminoalkoxide by means of the lithium amide of $N, N, N^{\prime}$-trimethylethanediamine $\mathbf{8 8 a}$, undergoes a regioselective addition of alkyllithiums on the $\mathrm{C}-\mathrm{C}$ double bond to give the benzyllithium intermediate $\mathbf{8 9}$. This was reacted diastereoselectively with several electrophiles, such as alkyl, allyl and benzyl halides, giving the $\alpha, \beta$-disubstituted 3-phenylpropanals 90, as $s y n$-isomers in good yields (49$78 \%$ ) and a good diastereoselectivity (90\% d.e.) (Scheme 29). ${ }^{47}$

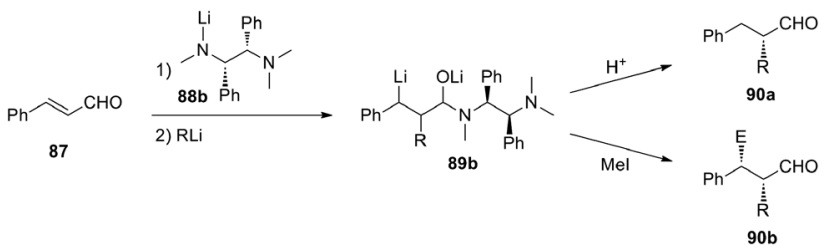

Scheme 30 Enantioselective carbolithiation-trapping reaction of 87.

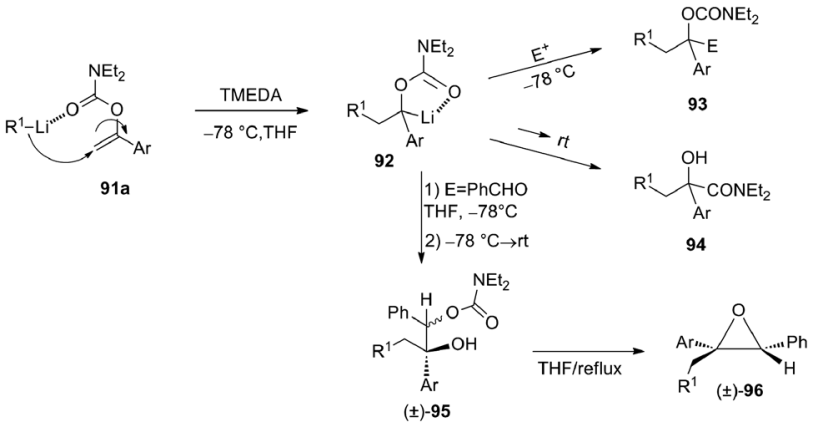

Scheme 31 One-pot tandem carbolithiation- $\alpha$-alkylation and -[1,2]Wittig rearrangement process of $\alpha$-aryl $O$-vinyl- $N, N^{\prime}$-diethyl carbamates $91 \mathrm{a}$ and synthesis of cis epoxide 96.

An asymmetric version of the above reported reaction was also developed. ${ }^{48}$ In the presence of the chiral lithium amides derived from $(R, R)$ or $(S, S)$-1,2-diphenyl- $N, N, N^{\prime}$-trimethylethanediamine (88b), it was possible to obtain an enantiomerically enriched organolithium intermediate. Subsequent hydrolysis or trapping with MeI led to $\alpha$-mono- or $\alpha, \beta$-disubstituted 3-phenylpropanals 90a or 90b with $76-96 \%$ e.e. (Scheme 30). An excess of chiral lithium amide ( 2 equivalent) is preferable because a lower amount provides similar yields but lower e.e. Contrary to the carbolithiation of cinnamyl derivatives by $\mathrm{RLi} /(-)-\mathbf{L 1}$, which has to be run in hydrocarbons, diethylether is the best solvent for this reaction. Moreover, primary, secondary and tertiary alkyllithiums can be used with good yields and enantioselectivities.

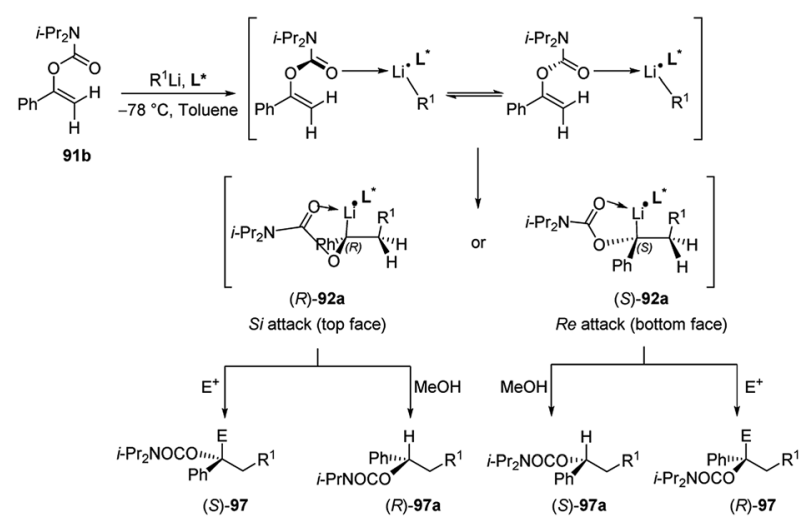

Scheme 32 Asymmetric carbolithiation-trapping reaction of 1-aryl-1alkenyl $N, N^{\prime}$-diisopropyl carbamates $91 \mathrm{~b}$. See Table 4 for ligands L* employed. 
Table 4 Asymmetric carbolithiation-trapping reaction of 1-aryl-1alkenyl $N, N^{\prime}$-diisopropyl carbamates 91 b

\begin{tabular}{|c|c|c|c|c|c|c|}
\hline $\mathrm{R}^{1} \mathrm{Li}$ & $\begin{array}{l}\text { Chiral } \\
\text { ligand }\left(\mathbf{L}^{*}\right)\end{array}$ & $\mathrm{E}$ & $\mathrm{E}^{+}$ & Product & $\begin{array}{l}\text { Yield } \\
(\%)\end{array}$ & $\begin{array}{l}\text { e.e. } \\
(\%)\end{array}$ \\
\hline$n$-BuLi & $(-)-\mathbf{L 1}$ & $\mathrm{MeOH}$ & $\mathrm{H}$ & $(S)-\mathbf{9 7} \mathbf{a}$ & 86 & 30 \\
\hline$n$-BuLi & $(-)-\mathbf{L} 2$ & $\mathrm{MeOH}$ & $\mathrm{H}$ & $(S)-97 \mathbf{a}$ & 63 & 58 \\
\hline$n$-BuLi & L4 & $\mathrm{MeOH}$ & $\mathrm{H}$ & $(S)-\mathbf{9 7 a}$ & 47 & 22 \\
\hline$n$-BuLi & L5 & $\mathrm{MeOH}$ & $\mathrm{H}$ & $(R)-97 \mathbf{a}$ & 81 & 20 \\
\hline$n$-BuLi & L6 & $\mathrm{MeOH}$ & $\mathrm{H}$ & $(S)-97 \mathbf{a}$ & 83 & 2 \\
\hline$n$-BuLi & L7 & $\mathrm{MeOH}$ & $\mathrm{H}$ & $(S)-97 \mathbf{a}$ & 73 & 16 \\
\hline i-PrLi & $(-)-\mathbf{L 1}$ & $\mathrm{CO}_{2}$ & $\mathrm{COOH}$ & $(R)-\mathbf{9 7} \mathbf{b}$ & 80 & 44 \\
\hline$t$-BuLi & $(-)-\mathbf{L 1}$ & $\mathrm{CO}_{2}$ & $\mathrm{COOH}$ & $(R)-97 \mathbf{b}$ & 77 & 24 \\
\hline
\end{tabular}

\subsection{Aryl vinyl carbamates}

In 1996 Snieckus and co-workers ${ }^{49}$ described an efficient carbolithiation of $\alpha$-aryl $O$-vinyl- $N, N^{\prime}$-diethyl carbamates 91 (Scheme 31). After the carbolithiation step, promoted by CIPE, the organolithium 92 was reacted with several electrophiles, obtaining trisubstituted aryl carbamates $\mathbf{9 3}$ or, after subsequent [1,2]-Wittig rearrangement, mandelic amides 94. When PhCHO was used as electrophile, a mixture of diastereomers of the product 95 is formed ( $3: 1$ by $\left.{ }^{1} \mathrm{H}-\mathrm{NMR}\right)$, and after heating at reflux an intramolecular cyclisation occurred, leading to isolation of only the epoxide $\mathbf{9 6}$ with cis relative stereochemistry.

Subsequently Hoppe and co-workers ${ }^{50}$ reported that also 1aryl-1-alkenyl $N, N^{\prime}$-diisopropyl carbamates 91b (Scheme 32) undergo intermolecular carbolithiation reactions easily. The syn addition of alkyllithium, in the presence of TMEDA, led to the formation of lithiated benzyl carbamates 92, which could be trapped by different electrophiles. Moreover, when the reaction was carried out in the presence of a chiral diamine, such as $(-)-\mathbf{L 1}$ or (-)-L2 (Fig. 1), moderate enantiofacial differentiation was observed (Scheme 32). ${ }^{50}$ The best results were obtained by using $n$-BuLi/(-)-L2 (58\% e.e.) and i-PrLi/(-)-L1 (44\% e.e.) (Table 4). This process can also provide a route to enantiomerically enriched benzyl alcohols. According to the asymmetric induction mechanism proposed by the authors ${ }^{50}$ the diastereoisomeric complexes, formed by the coordination between the alkyllithium and the chiral ligand, react with the double bond in an intramolecular syn-addition to give the enantiomeric benzyllithium derivatives $(R)$-92a and $(S)$-92a. They are configurationally stable and can be trapped with electrophiles. In particular, it was observed that the lithium/ hydrogen exchange occurs with retention of configuration, giving the enantiomeric products $(R)-\mathbf{9 7 a}$ and $(S)-\mathbf{9 7 a}$. On the contrary, using others electrophiles, an inversion of the configuration was observed (Scheme 32).

The carbolithiation of the stilbene carbamates $(Z)-98$ and $(E)$ 98 in the presence of TMEDA was also investigated (Scheme 33). In this case the carbolithiation of the 1,2-disubstituted olefins led to the formation of two new contiguous stereogenic centres. Notably, $(E)-98$ do not undergo the addition of $n$-BuLi/TMEDA, instead it provides a $\beta$-elimination which gives rise to diphenylethyne in high yield. This highlight that the outcome of the
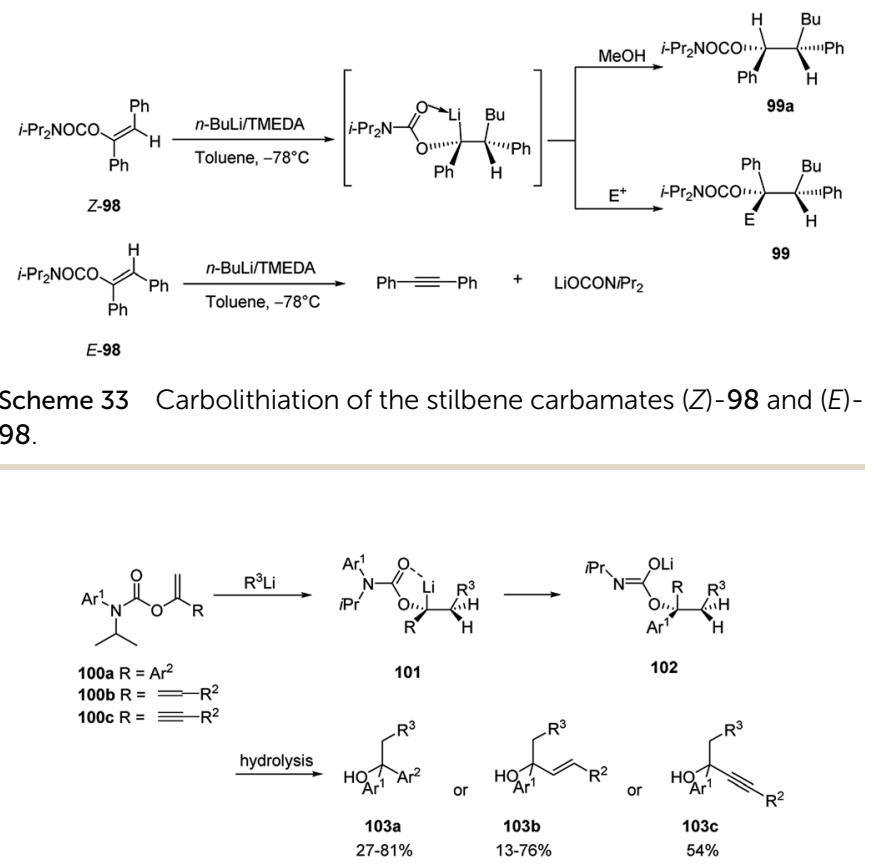

Scheme 34 Carbolithiation of aryl, alkenyl and alkynyl enol carbamates bearing an $\mathrm{N}$-aryl substituent $100 \mathrm{a}-\mathrm{c}$.

carbolithiation on stilbene derivatives is strictly depending on the stereochemistry of the starting material.

Clayden and co-workers ${ }^{51}$ described the carbolithiation of aryl, alkenyl and alkynyl enol carbamates bearing an $\mathrm{N}$-aryl substituent 100a-c (Scheme 34). After the syn addition of the alkyllithium to the vinyl double bond, in the presence of $N, N^{\prime}$ dimethylpropyleneurea (DMPU), the resulting organolithium carbamate intermediate (101) rearranges with $\mathrm{N}-\mathrm{C}$ migration of the $\mathrm{N}$-aryl substituent, creating a novel quaternary carbon centre $\alpha$ to oxygen (in 102).

Such migration occurs with inversion of configuration. The products obtained can be hydrolysed to give benzyl, allyl and propargyl branched tertiary alcohols 103a-c by means of a onepot tandem process. Additionally, $N$-alkenyl carbamates 104 have been shown to be useful substrates for carbolithiation with primary, secondary, and tertiary alkyllithium reagents (Scheme $35 a){ }^{52}$ In an attempt to induce stereoselectivity in the

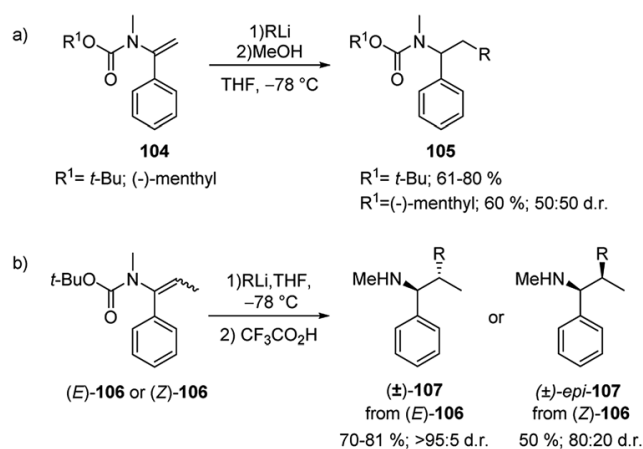

Scheme 35 Carbolithiation-trapping reaction of $\mathrm{N}$-alkenyl carbamates 104 (route a) and (route b) 106. 

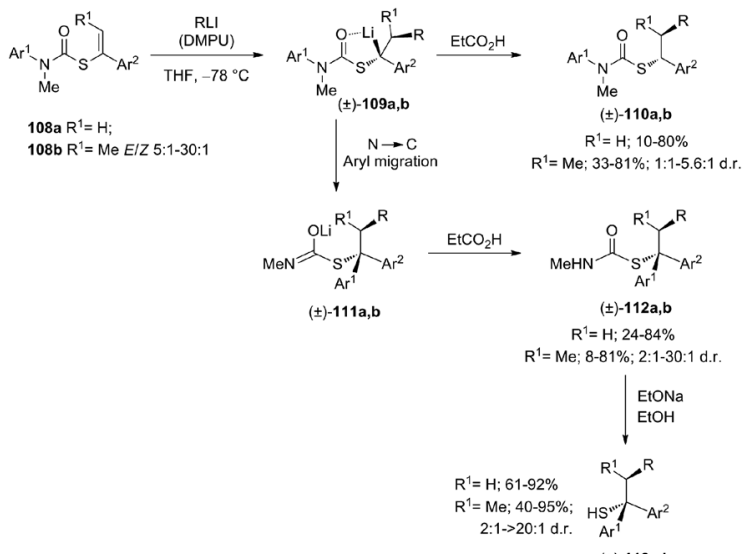

$( \pm)-113 a, b$

Scheme 36 Carbolithiation of S-alkenyl- $N$-arylthiocarbamates 108

carbolithiation process the (-)-menthyl carbamate was employed as substrate. However, no diastereoselectivity was observed. $N$-t-butoxycarbonyl propenyl carbamates 106 were also carbolithiated, protonated and deprotected in a one-pot synthesis of amines (Scheme 35b). ${ }^{52}$ While $E-\mathbf{1 0 6}$ provided $( \pm)$-107 in high 95 : 5 d.r. in only 1 hour, the $Z$ isomer of the $\beta$ substituted vinyl carbamate 106 resulted less reactive providing the carbolithiation product after 24 hours and, after hydrolysis, the epi-107 diastereoisomer in lower 80:20 d.r. According to the authors, the loss of diastereospecificity was explained by the long reaction time during which the syn-carbolithiation is followed by a partial epimerisation of the organolithium intermediate.

The carbolithiation of $S$-alkenyl- $N$-arylthiocarbamates 108 with a range of alkyllithium reagents, stereospecifically generates benzyllithium intermediates $\mathbf{1 0 9}$, which may also undergo intramolecular arylation, due to the $\mathrm{N}$ to $\mathrm{C}$ aryl migration, providing tertiary thiocarbamates $\mathbf{1 1 2}$. The latter are in turn precursors of new families of tertiary functionalised thiols 113, obtained in many cases with diastereoselective control (Scheme 36). ${ }^{53}$

The first asymmetric version of the carbolithiation of $S$-alkenyl$\mathrm{N}$-arylthiocarbamates (108a) was also developed (Scheme 37). ${ }^{54}$ The addition of $n$-BuLi to alkenylthiocarbamates in the presence of chiral diamine ligands, such as $(-)-\mathbf{L 1}$ or $(+)$-sparteine surrogate (L3), followed by protonation, generates enantiomerically enriched thiocarbamate derivatives of secondary thiols.

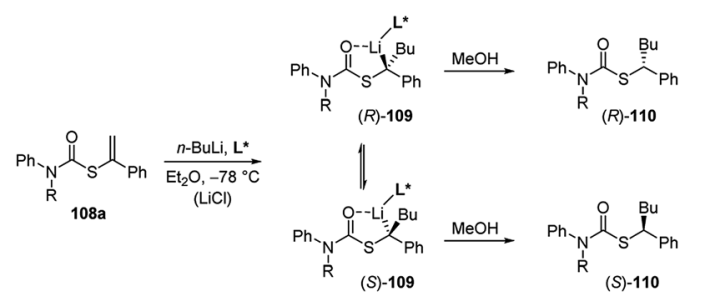

$L^{\star}=L 1(R) 34 \%$ e.e.; (S) $24 \%$ e.e. (LiCl); L3 (R) $68 \%$ e.e.; L9 (R) $20 \%$ e.e.; L10 $0 \%$ e.e.

Scheme 37 Asymmetric carbolithiation of S-alkenyl- $N$-arylthiocarbamates (108a). See Fig. 1 for ligands L* structures.

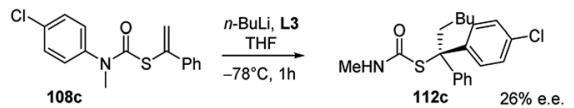

Scheme 38 Enantioselective carbolithiation/rearrangement process of $S$-alkenyl- $N$-arylthiocarbamates.

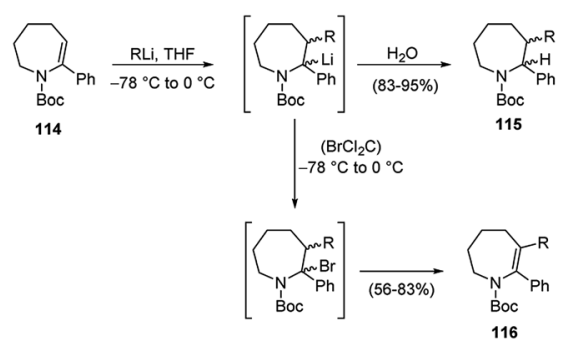

Scheme 39 Carbolithiation of the double bond of a cyclic enecarbamate 114 .

Remarkably, the two pseudoenantiomeric chiral ligands give the same $(R)$ enantiomer of the product. However, when $\mathrm{LiCl}$ is added to the carbolithiation mediated by $(-)-\mathbf{L 1}$, an inversion of the asymmetric induction was observed, providing the opposite $(S)$ enantiomer.

In order to explain these results the authors proposed two possible reaction pathways. In the first one, kinetic control induces facial selectivity in the addition step to give a configurationally stable organolithium-ligand complex, the stereospecific protonation of which yields to enantiomerically enriched compounds. In the second pathway, the addition step may or may not be enantioselective, but thermodynamic control over the equilibration of the resulting diastereoisomeric organolithium complexes gives enantiomerically enriched products, again by stereospecific protonation. Moreover, by using THF as solvent and L3 as chiral ligand, a tandem enantioselective carbolithiation/rearrangement process, due to an in situ $\mathrm{N}-\mathrm{C}$ aryl migration, occurs, leading to enantiomerically enriched tertiary thiols precursors (Scheme 38).

The first carbolithiation of the double bond of a cyclic enecarbamate $\mathbf{1 1 4}$ was reported by Coudert and co-workers (Scheme 39). ${ }^{55}$ Throughout this reaction it is possible to obtain either saturated or unsaturated polysubstituted nitrogen-containing heterocycles. Under the reaction conditions used, allylic protons were not affected and the expected regioselective addition of $\mathrm{RLi}$ to the $\mathrm{C}-3$ position of the substrate was observed.

The carbolithiation of acyclic ene-carbamates 117 was also developed (Scheme 40) ${ }^{56}$ This process allows an easy access to $\alpha$-amino acids bearing a quaternary carbon next to the nitrogen. After the nucleophilic intermolecular addition of alkyllithium reagent to double bond of the ene-carbamate, by warming the reaction mixture from $-78{ }^{\circ} \mathrm{C}$ to $0{ }^{\circ} \mathrm{C}$, the benzylic organolithium intermediate became thermally labile and spontaneously underwent an unexpected internal Boc-carbonyl migration from the nitrogen to the benzylic carbon to give the lithium amide 118. Subsequent hydrolysis provided in good 

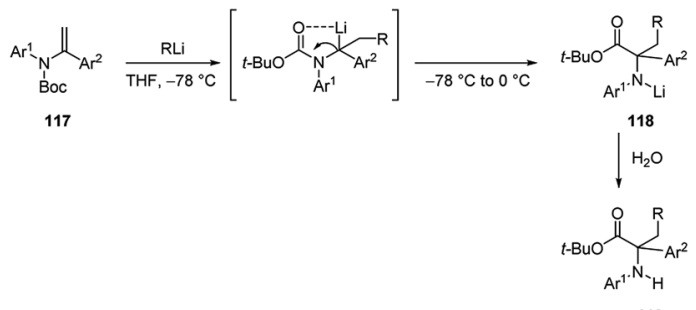

119

Scheme 40 Carbolithiation of acyclic ene-carbamates 117.

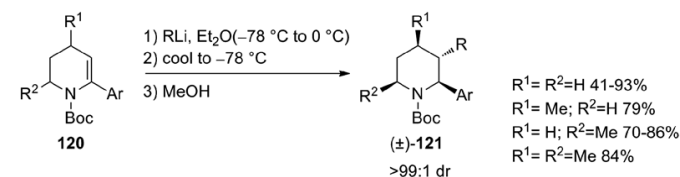

Scheme 41 Diastereoselective carbolithiation and protonation of 120

yields (14-89\%) $\alpha$-amino esters 119, precursors of non-natural $\alpha$-amino acids having a quaternary $\alpha$-carbon centre.

The first example of highly diastereoselective carbolithiations, followed by protonation, of $\alpha$-arylated dehydro-piperidine enecarbamates $\mathbf{1 2 0}$ with alkyllithium nucleophiles was also described (Scheme 41). ${ }^{57}$ This umpolung-type synthetic sequence can lead to the diastereoselective synthesis of piperidine derivatives bearing vicinal $(\mathrm{C} 2, \mathrm{C} 3)$ substituents, providing a method to obtain difunctionalized piperidines, useful precursors for the synthesis of compounds of medicinal and biological importance. The carbolithiation process is highly diastereoselective, with the C-2 aryl and the C-3 alkyl groups in anti position. The nature of the aryl substituent has a marked effect on the extent of competing nucleophile addition into the Boc group. In fact, enecarbamates bearing electron-rich arenes as well as sterically hindered like 1-naphthyl, mainly undergo addition of the alkyllithium to the Boc group.

The diastereoselective carbolithiation of the piperidine enecarbamate bearing other substituents on the ring was also explored. When, after introduction of the alkyllithium at $-78{ }^{\circ} \mathrm{C}$, the reaction mixture was warmed at $-30{ }^{\circ} \mathrm{C}$ in the presence of hexamethylphosphoramide (HMPA), the desired vicinally functionalized piperidines $\mathbf{1 2 1}$ were obtained in higher yields with respect to the additive-free conditions. ${ }^{58}$ In fact, without additive, the attack on the Boc-group by strongly nucleophilic alkyllithiums is competitive with $\beta$-alkylation. Moreover, piperidine-derived tertiary benzylic organolithiums, generated by carbolithiation of $\alpha$-aryl piperidine enecarbamates, can be successfully trapped, under HMPA-mediated

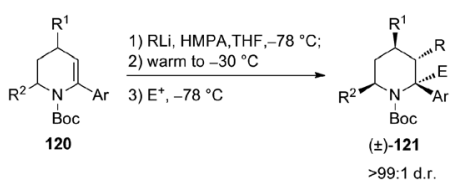

Scheme 42 Carbolithiation and electrophilic trapping of 120 .

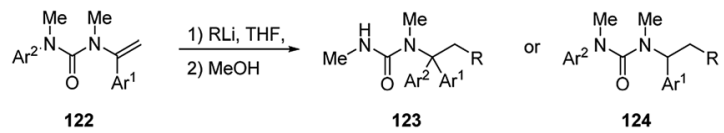

Scheme 43 Carbolithiation of $N$-alkenyl ureas 122

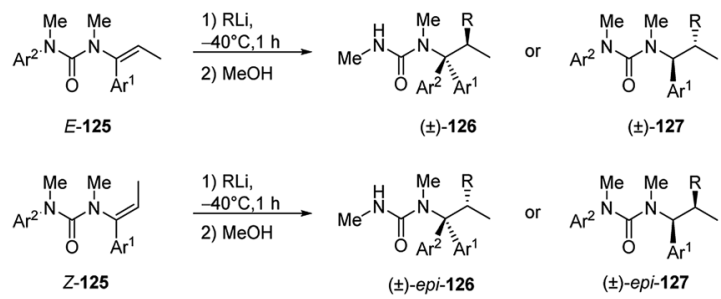

Scheme 44 Carbolithiation of $\beta$-substituted vinyl ureas (125).

conditions, with several carbon electrophiles, (Scheme 42). ${ }^{58}$ Such carbolithiation/trapping process allowed to obtain, in diastereoselective manner, vicinally functionalized piperidines bearing $\alpha$-amino quaternary stereocenters with up to three contiguous stereocenters.

A proximity effect similar to the carbamoyl group is provided by the 2-pyridyloxy group, which directs the alkyllithiums addition to the double bond of the enol ether $\alpha$-(2-pyridyloxy) styrene. After the carbolithiation step, the resulting $\alpha$-(2-pyridyloxy)benzyllithium undergoes an anionic rearrangement due to the migration of the pyridyl group affording tertiary pyridyl carbinols. ${ }^{59}$

\subsection{Aryl vinyl ureas}

$\mathrm{N}$-Alkenyl ureas 122 also exhibited Umpolung reactivity undergoing facile addition of alkyllithium at their $\beta$-carbons (Scheme 43). ${ }^{52}$ When the reaction was performed in THF at $-78^{\circ} \mathrm{C}$ or, more easily, by using hindered alkyllithium, the carbolithiation products 124 were obtained in moderate to good yields (47$77 \%) .{ }^{52}$ The carbolithiation reaction of $N$-alkenyl ureas bearing an $\mathrm{N}$-aryl substituent in THF at $-50{ }^{\circ} \mathrm{C}$ was coupled with $\mathrm{N} \rightarrow \mathrm{C}$ aryl transfer within the lithiated urea intermediate, giving rise to rearranged products $\mathbf{1 2 3}$, in good to excellent yields (72$96 \%) .{ }^{60}$ Migration of a phenyl ring is generally faster and cleaner than migration of other substituted aryl groups, such as the electron-rich ones, and using less hindered alkyllithium. This tandem $\beta$-alkylation- $\alpha$-arylation protocol allows the construction in a single pot of two new $\mathrm{C}-\mathrm{C}$ bonds at $\alpha$ and $\beta$ carbons of the urea-substituted alkene and at the same time it shows the ability of urea moieties to promote carbolithiation by CIPE.

In the case of $\beta$-substituted vinyl ureas (Scheme 44$),{ }^{52,60}$ the addition of alkyllithium to $(E)$-isomer $\left((E)\right.$-125) at $-40{ }^{\circ} \mathrm{C}$ in toluene, followed by protonation with retention of configuration, led to the formation of products 126, as single diastereomers. In order to suppress rearrangement a noncoordinating solvent was used.

The tandem carbolithiation/protonation process is completely diastereospecific. In fact, using the (Z)-125 as starting material, under the same reaction conditions, the other 


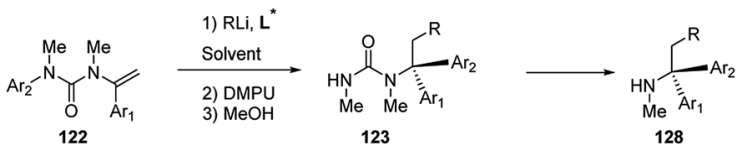

$L^{*}=L 1=30-88 \%$ e.e.; $L 3=6-90 \%$ e.e.; $L 4=0 \%$ e.e.; $L 8=0 \%$ e.e.

Scheme 45 Enantioselective carbolithiation of $N$-alkenyl- $N^{\prime}$-arylureas 122 .

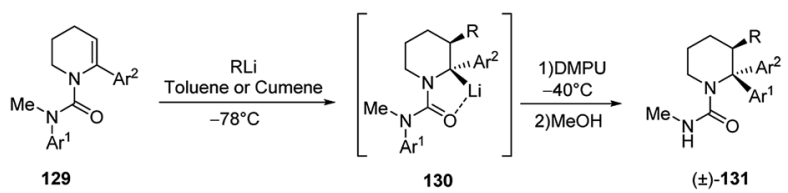

Scheme 46 Carbolithiation/rearrangement sequence of cyclic ureas 129

diastereomer epi-127 is selectively obtained. When the carbolithiation of $\beta$-methyl vinyl ureas, $(E)-\mathbf{1 2 5}$ or $(Z)-\mathbf{1 2 5}$, was carried out in THF, a more coordinating solvent, or in toluene and in the presence of DMPU as coordinating co-solvent, a rearrangement occurred, due to the $\mathrm{N} \rightarrow \mathrm{C}$ aryl migration, giving 126 or epi-126 compounds. Contrary to as shown before for the $O$-vinyl carbamates, the migration of the $N$-aryl groups in $N$-alkenyl ureas occurred with retention of configuration. Both the carbolithiation and aryl migration steps are then stereospecific, since changing the stereochemistry of the double bond in the starting material, the relative configuration of the products changes.

In the presence of chiral diamine ligands, such as (-)-L1 and L3, enantioselective carbolithiation of $N$-alkenyl- $N^{\prime}$-arylureas (122) occurred, leading to benzyl organolithiums which, in the presence of DMPU, undergo rearrangement with the $\mathrm{N} \rightarrow \mathrm{C}$ aryl transfer, providing optically active 123 (Scheme 45). ${ }^{61}$ The stereochemical outcome of the reaction depends on the ligand employed.

The tandem asymmetric carbolithiation/rearrangement process allows to generate urea derivatives of enantiomerically enriched $\alpha, \alpha$-diarylamines $\mathbf{1 2 8}$. In the asymmetric version, the aryl migration is also stereochemically retentive.

The carbolithiation/rearrangement sequence was also reported for cyclic ureas (Scheme 46). ${ }^{62}$ As a matter of fact, the urea derivatives of tetrahydropyridines $\mathbf{1 2 9}$ undergo addition of alkyllithium on the endocyclic double bond. By adding DMPU and warming the reaction mixture, the organolithium intermediate 130, formed after the carbolithiation step, undergo an efficient rearrangement, due to the migration of the $\mathrm{N}$-aryl group to the lithiated centre. The whole process allowed to synthetized 2,2-diaryl-3-alkyl piperidines 131 in about $60 \%$ yield as single diastereoisomer.

\subsection{Alkynes}

Carbolithiation of alkynes enables to prepare tri- and tetrasubstituted alkenes in a stereoselective manner. Nevertheless the versatility of this method is fairly limited by the nature of

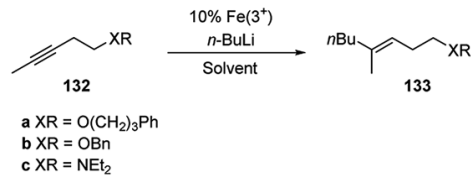

Scheme 47 Carbolithiation of alkynes bearing an alkoxy or amino group 132

Table 5 Carbolithiation of $132 a-c$ in the presence of catalytic amounts of these Fe(III) complexes and subsequent acid hydrolysis

\begin{tabular}{|c|c|c|c|c|}
\hline Substrate & Fe salt & Solvent & $T\left({ }^{\circ} \mathrm{C}\right)$ & Yield (\%) \\
\hline $132 a$ & $\mathrm{FeCl}_{3}$ & THF & -40 & 0 \\
\hline $132 a$ & $\mathrm{FeCl}_{3}$ & $\mathrm{Et}_{2} \mathrm{O}$ & -40 & $79(\mathbf{1 0 6} \mathbf{)})$ \\
\hline $132 a$ & $\mathrm{FeCl}_{3}$ & Benzene & -20 & $86(106 a)$ \\
\hline $132 a$ & $\mathrm{FeCl}_{3}$ & Toluene & -20 & $97(\mathbf{1 0 6 a})$ \\
\hline $132 b$ & $\mathrm{FeCl}_{3}$ & $\mathrm{Et}_{2} \mathrm{O}$ & -40 & $79(\mathbf{1 0 6} b)$ \\
\hline $132 b$ & $\mathrm{Fe}(\mathrm{acac})_{3}{ }^{a}$ & Toluene & -20 & $97(\mathbf{1 0 6} b)$ \\
\hline $132 b$ & $\mathrm{Fe}(\mathrm{dbm})_{3}{ }^{a}$ & Toluene & -20 & $85(106 b)$ \\
\hline $132 b$ & $\mathrm{FeCl}_{3}$ & Toluene & -20 & $13(\mathbf{1 0 6} b)$ \\
\hline $132 \mathrm{c}$ & $\mathrm{Fe}(\mathrm{acac})_{3}$ & Toluene & -20 & $72(106 c)$ \\
\hline
\end{tabular}

the substrate. ${ }^{63}$ Indeed, in general, alkynes cannot be carbolithiated under the usual conditions because they are deprotonated at the alkyne carbon, if terminal, or at the propargylic positions. ${ }^{\mathbf{1 5}}$ Moreover, in reactions of alkynes without a conjugated electron-withdrawing group, the regioselectivity becomes the principal problem. Another problem may be the stereochemistry because of the easy isomerization of the vinyllithium intermediates. ${ }^{63}$

In 2001 Hojo et al. ${ }^{63}$ reported that iron salts, such as $\mathrm{FeCl}_{3}$, $\mathrm{Fe}(\mathrm{acac})_{3}(\mathrm{acac}=$ acetylacetone $)$ and $\mathrm{Fe}(\mathrm{dbm})_{3}(\mathrm{dbm}=$ dibenzoylmethane), catalyze the regio- and stereoselective carbolithiation of alkynes bearing an alkoxy or amino group (Scheme 47). Therefore, the addition of $n$-BuLi on the substrates 132a-c in the presence of catalytic amounts of these $\mathrm{Fe}(\mathrm{III})$ complexes affords, after acid hydrolysis, the corresponding butylated products $(\mathbf{1 3 3 a}-\mathbf{c})$ as single $(E)$-isomers in good to excellent yields (Table 5). The choice of the solvent is crucial for this reaction since in benzene or toluene, the butylated products were obtained in high yields, whereas in THF the desired product was not formed.

The carbolithiation of $\mathbf{1 3 2} \mathbf{b}$ in the best conditions (Fe(acac) $)_{3}$ and toluene in which the iron salt is soluble) followed by the treatment of the vinyllithium intermediate (134) with several electrophiles, such as $\mathrm{SiMe}_{2} \mathrm{HCl}$, PhCHO, EtCHO, PhCOEt, gave the products 135a-e in stereoselective manner and in good yields (Scheme 48).

Tomooka and co-workers ${ }^{64}$ described the carbolithiation reaction of unsymmetrical dialkynes $\mathbf{1 3 6}$ with a tertiary alcoholic function in propargylic position and bearing TBDPS (tertbutyldiphenylsilyl) and TIPS (triisopropylsilyl) protecting groups at the $\gamma$ and $\gamma^{\prime}$ position respectively. Thus, the addition of $n$-BuLi to the propargylic alcohol 136 in the presence of TMEDA, followed by protonation, gave the allylic alcohol 138 


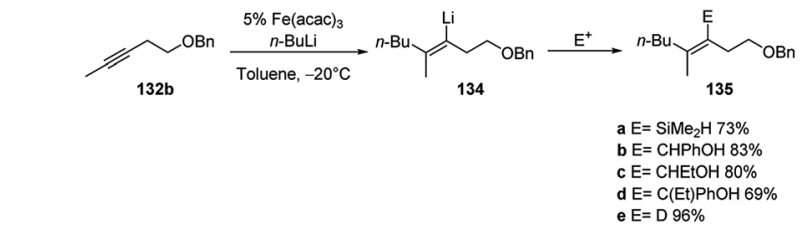

Scheme 48 Carbolithiation and electrophilic trapping of 132b

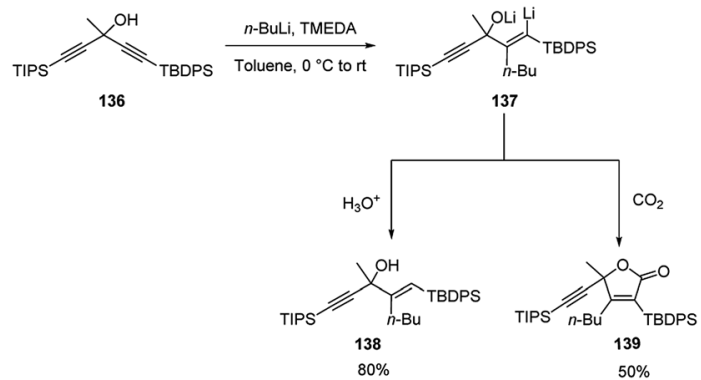

Scheme 49 Carbolithiation reaction of unsymmetrical dialkynes 136.

with a $(E)$-configured trisubstituted alkene moiety in $80 \%$ yield. When the vinyllithium intermediate 137 was treated with $\mathrm{CO}_{2}$ as electrophile, the $\gamma$-lactone $\mathbf{1 3 9}$ was instead obtained in $50 \%$ yield (Scheme 49).

The carbolithiation reaction occurs with excellent regioselectivity at the TBDPS alkyne, since the TBDPS, rather than the TIPS group, acts as an activating group for the alkynyl moiety, despite its steric disadvantage. The origin of this TBDPS effect has been attributed to a stereoelectronic effect of the phenyl group on silicon, which increases the electrophilicity of the $\beta$ carbon atom on the alkynyl group due to hyperconjugation of the phenyl and alkyne $\pi$ orbitals through the Si-C $\sigma^{*}$ bond. These results show that an arylsilyl group can act not only as a protecting group but also as an activating group of the alkynyl moiety, providing access to a strategy by which it is possible to synthesize multifunctionalized alkenes. ${ }^{64}$

Carbolithiation reaction of diphenylacetylene (140) was also exploited as the key synthetic step for the highly stereoselective preparation of $(Z)$-tamoxifen, a therapeutic agent for the treatment of the estrogen-dependent breast cancer. ${ }^{65}$ The syn-addition of alkyllithium to the triple bond of $\mathbf{1 4 0}$ led to the vinyllithium intermediate $(Z)-\mathbf{1 4 1}$, which, under the reaction conditions, rapidly isomerizes generating the thermodynamically more stable vinyllithium species $(E)$-141 with a transdiphenyl geometry. Subsequent protonation with $\mathrm{MeOH}$ of the lithiated $(E)$-alkene led to the 1,1,2-trisubstituted trans alkene 142, while the trapping with triisopropylborate $\left(\mathrm{B}(\mathrm{O} i \mathrm{Pr})_{3}\right)$ as electrophile, yielded the $(E)$-vinyl boronic acids 143, which can undergo the further transformation to (Z)-tamoxifen and related analogues by Pd-catalyzed Suzuki-Miyaura crosscoupling reaction with the appropriate aryl iodide (Scheme 50).

Recently, a similar synthesis of (Z)-tamoxifen was also reported by Feringa and co-workers through a two-step procedure involving the carbolithiation of diphenylacetylene (140) followed by the direct cross-coupling of the alkenyllithium

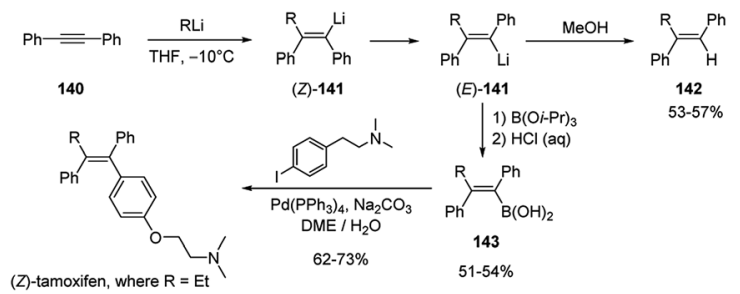

Scheme 50 Carbolithiation of 140 and synthesis of (Z)-tamoxifen.

intermediate (141, where $\mathrm{R}=\mathrm{Et}$ ) with the suitable bromide in the presence of a highly active palladium nanoparticle catalytic system, thus avoiding the transmetalation step. ${ }^{66}$

In the carbolithiation of highly strained acetylenic bonds, just like those of 5,6,11,12-tetradehydrodibenzo[a,e]cyclooctene (144), ${ }^{67}$ nucleophilic addition of RLi to the triple bond smoothly occurs presumably as a consequence of a thermodynamic advantage gained by release of the strain upon sp-to-sp ${ }^{2}$ hybridization change. The resulting vinylic anions 145 spontaneously underwent trans-annular cyclization, providing the tetracyclic organolithium intermediate 146, which can be in situ trapped by various electrophiles to finally give dibenzo $[b, f]$ pentalenes $\mathbf{1 2 0}$.

Various aldehydes were employed as electrophiles, such as benzaldehyde, $p$-chloro- and $p$-nitrobenzaldehyde, 2-naphthaldehyde, and heptanal. Also, cyclohexenone, PhCOCl, MeI, and $\mathrm{Me}_{3} \mathrm{SiCl}$ were used as electrophiles, affording the desired products 147 in moderate to good yields (Scheme 51).

As reported above, in the carbolithiation reaction of alkynes the deprotonation of both acetylenic and propargylic protons generally predominates over the addition of alkyllithium to the triple bond. However, carbolithiation of alkynes having propargyl protons is possible when a heteroatom directing group on alkyne moiety, such as alkoxy or amino group, facilitate the addition reaction through intramolecular coordination ${ }^{63}$ and in the presence of iron-copper catalysts. ${ }^{68}$ The reaction conditions optimized ( $\mathrm{Fe}(\mathrm{acac})_{3}$ and toluene) by Hosomi and co-workers ${ }^{63}$ for carbolithiation of heteroatom-containing alkynes were found to be not effective for the addition to aryl alkyl acetylenes like 148. On the contrary the use of $\mathrm{FeCl}_{3}$ as catalyst and $\mathrm{Et}_{2} \mathrm{O}$ as solvent, worked much better, providing the desired 1,1,2trisubstituted alkenes (151 and 152) in moderate to excellent

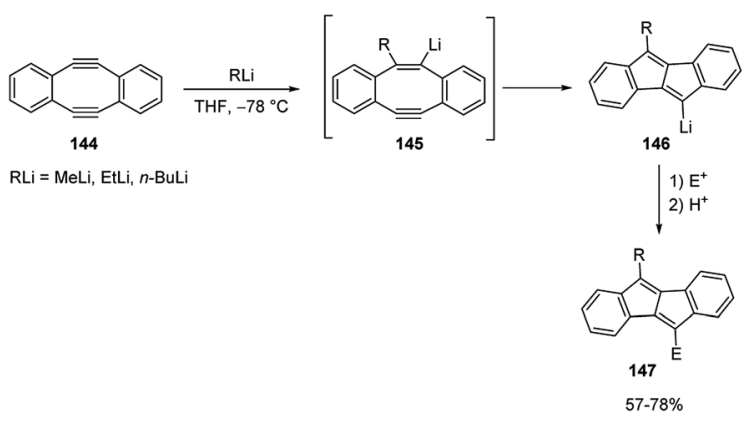

Scheme 51 Carbolithiation and electrophilic trapping of 144. 


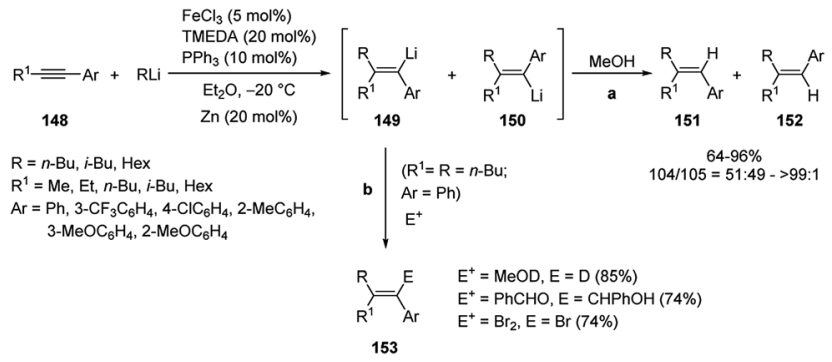

Scheme 52 Iron-catalyzed carbolithiation of alkynes 148

yields (64-96\%). ${ }^{68}$ It was also found that using TMEDA and $\mathrm{PPh}_{3}$ as additives increased the yield. Even when the stereoselectivity was low, the isomer 151 predominated over 152, showing that the initial product was the syn-adduct $\mathbf{1 4 9}$, which isomerizes to 150 under the reaction conditions. Moreover, if the carbolithiation reaction was performed in the absence of an iron catalyst, no carbolithiated products was formed. Since a certain amount of $\mathrm{RLi}$ was consumed for the reduction of $\mathrm{FeCl}_{3}$ to a catalytically active low valent species, the addition of zinc (20 mol\%), which acts as reducing agent in place of alkyllithium, increased the yield in some cases. Furthermore, when $\mathrm{R}=\mathrm{R}^{1}$, an excess alkyllithium to alkyne was used, whereas in the case of $R \neq R^{1}$, an excess of alkyne to alkyllithium in combination with zinc as a reductant, was used in order to minimize the $E / Z$ isomerization. Then the carbolithiation step was followed by protonation with $\mathrm{MeOH}$ (Scheme 52, pathway a). The vinyllithium intermediate $\mathbf{1 4 9}$, formed by the addition of $n$-BuLi to 1-phenyl-1-hexyne $\left(\mathbf{1 4 8}, \mathrm{R}^{1}=n\right.$ - $\left.\mathrm{Bu}, \mathrm{Ar}=\mathrm{Ph}\right)$, was also reacted with several electrophiles, such as MeOD, benzaldehyde and 1,2-dibromoethane, leading to tetrasubstituted alkenes 153 in good yield (74-85\%) (Scheme 52, pathway b). The catalytic system described above was not successful in the carbolithiation reaction performed with aryllithium reagents like 154, where, instead, the $\mathrm{Fe}-\mathrm{Cu}$ cooperative catalysis worked.

Thus, the reaction of phenyllithium with 1-phenylpropyne (154a) in the presence of $\mathrm{Fe}(\mathrm{acac})_{3}(5 \mathrm{~mol} \%), \mathrm{CuBr}(10 \mathrm{~mol} \%)$ and $\mathrm{PBu}_{3}(40 \mathrm{~mol} \%)$ in $\mathrm{Et}_{2} \mathrm{O}$ at $30{ }^{\circ} \mathrm{C}$ gave $62 \%$ yield of $(E)-1,2-$ diphenylpropene (155a) and its stereo- and regioisomers 156a and 157a in $93: 5: 2$ ratio (Scheme 53). The aryllithiation proceeded in high stereo- and regioselectivity also between 3,5xylyllithium and 1-phenyl-1-octyne (154b). ${ }^{63}$

Only a few examples of carbolithiation of aryllithiums to unsaturated compounds such as enynes have been reported in the literature. Presumably, because at low temperature the

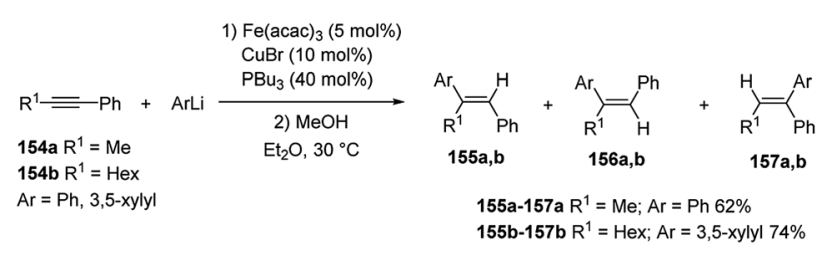

Scheme 53 Iron-catalyzed carbolithiation of alkynes 154 using aryllithium.

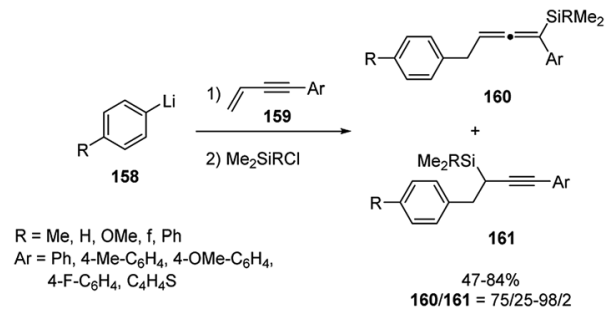

Scheme 54 Carbolithiation of conjugated enynes 159 with aryllithiums and synthesis of allenylsilanes 160 .

addition of aryllithiums to a $\mathrm{C}-\mathrm{C}$ unsaturated bond is quite slow, whereas at higher temperature the resulting organolithium compound reacts with the butyl halide formed in the halogen-lithium exchange reaction employed to obtain in situ the aryllithium reagent. This side reaction obviously competes the subsequent reaction with an electrophile. Recently, Yoshida and co-workers developed the carbolithiation of conjugated enynes with aryllithiums, followed by electrophilic substitution with chlorosilanes, using an integrated microflow system. Such approach, relying on the use of flow microreactors to control extremely fast reactions and named "flash chemistry", allows to carry on many chemical reactions practically impossible in batch macroreactor ${ }^{69}$ Throughout carbolithiation performed in such flow microreactors, they obtained various allenylsilanes 160 in a regioselective manner and in good yields (47-100\%) (Scheme 54). In fact, the use of a microflow system allows to perform the carbolithiation at higher temperature, such as 0 or $20{ }^{\circ} \mathrm{C}$, avoiding the side reaction with the butyl halide encountered in conventional macrobatch conditions. It was found that the conversion and the yields significantly depend on both temperature and residence time in the microreactors. In particular, precise residence time control was considered responsible for the success of the transformation.

The methodology resulted efficient and versatile with several aryllithium compounds 158, both having electron-donating and electron-withdrawing groups, as well as with various 4-aryl-but1-en-3-ynes and 4-heteroaryl-but-1-en-3-yne 159. Several silanes, such as chlorotrimethylsilane, chlorodimethylsilane, chlorodimethyl(vinyl)silane, and allylchlorodimethylsilane were also used as electrophiles. ${ }^{70}$

Still by means of a flow microreactor system, Yoshida and coworkers reported the synthesis of enantioenriched chiral allenes via asymmetric carbolithiation of conjugated enynes followed by quench with electrophiles. ${ }^{71}$ The asymmetric carbolithiation of conjugated enynes 162 in the presence of the chiral ligand (-)-L1 leads to the formation of chiral organolithium intermediates 163, which can be trapped with electrophiles providing the chiral allenes 164, useful and versatile building blocks. This process is usually very difficult to achieve with conventional methods because the organolithium intermediates 163 are configurationally unstable and rapidly epimerize before reacting with electrophiles. On the contrary, in a flow microreactor system, endowed with high-resolution control of the residence time, this problem can be overcome, thus enabling to react the configurationally unstable chiral 


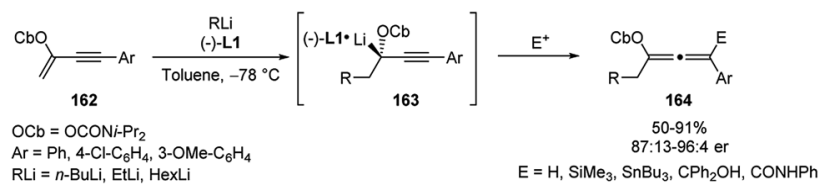

Scheme 55 Asymmetric carbolithiation of conjugated enynes 162 and synthesis of 164 .

organolithium intermediates with electrophiles before their epimerization. Thus, by flash chemistry approach, the alkyllithium addition to conjugated enynes bearing an appropriate carbamoyloxy $(\mathrm{OCb})$ directing group and in the presence of (-)-L1 allows reaction of the chiral organolithium 163 which several electrophiles, such as $\mathrm{MeOH}, \mathrm{Me}_{3} \mathrm{SiCl}, \mathrm{Bu}_{3} \mathrm{SnCl}$, benzophenone, and phenyl isocyanate, providing the corresponding chiral allenes in highly enantioselective manner and moderate to high yields (50-91\%) (Scheme 55). ${ }^{71}$

Better results were obtained by optimization of reaction conditions. In fact, increasing residence time and temperature the yield increases while the enantiomeric composition decreases, presumably because of epimerization of the intermediate 163. Higher yield and enantioselectivity were achieved within a small residence time-temperature domain with a residence time of $25 \mathrm{~s}$ and temperature of $-78^{\circ} \mathrm{C}$. $^{71}$

The carbolithiation of benzyne (168) with a functionalized aryllithiums, followed by electrophiles trapping in an integrated flow microreactor system was also reported. ${ }^{72}$

It should be noted that such a transformation may be very difficult or impossible in batch because of short lifetimes of the highly reactive lithiated intermediates. Therefore, $o$-bromophenyllithium (167) and $p$-chlorophenyllithium (169), generated in situ by halogen/lithium exchange of 1-bromo-2iodobenzene (165) and 1-bromo-4-chlorobenzene (166) respectively, were mixed at $-70{ }^{\circ} \mathrm{C}$ in a microreactor. The aryllithium 167 selectively decomposed to generate 168 without affecting the functionalized aryllithium 169 , which at $-30{ }^{\circ} \mathrm{C}$ spontaneously added to 168 . The resulting biaryllithium 170 eventually reacted with electrophiles in a subsequent microreactor to provide the corresponding three-component coupling products 171 (Scheme 56). Also, in this case, the fine tuning of reaction conditions, more specifically temperature and residence time, is responsible for the success of the multi-step reaction. Again, several electrophiles such as MeOTf, TMSOTf, phenyl

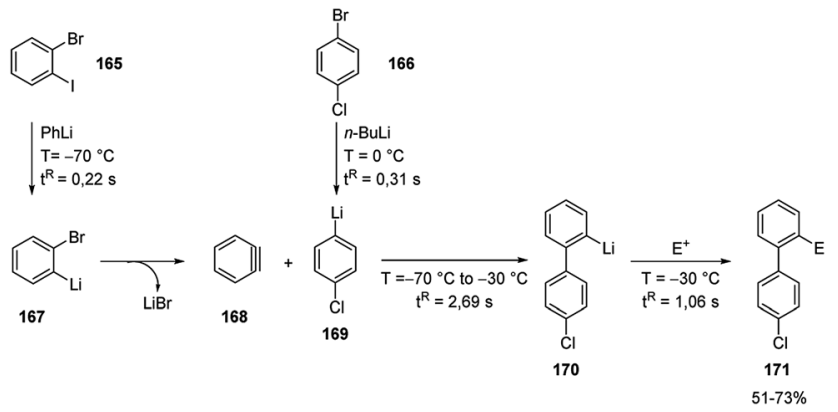

Scheme 56 Carbolithiation of benzyne (168) with functionalized aryllithiums followed by reactions with electrophiles.

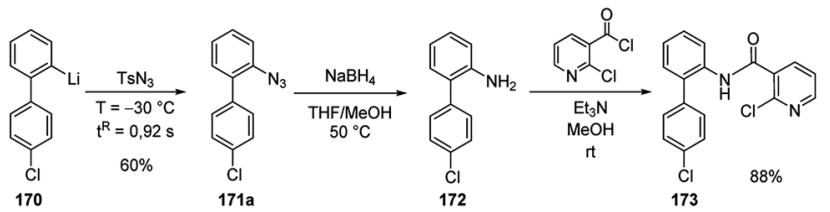

Scheme 57 Synthesis of boscalid (173).

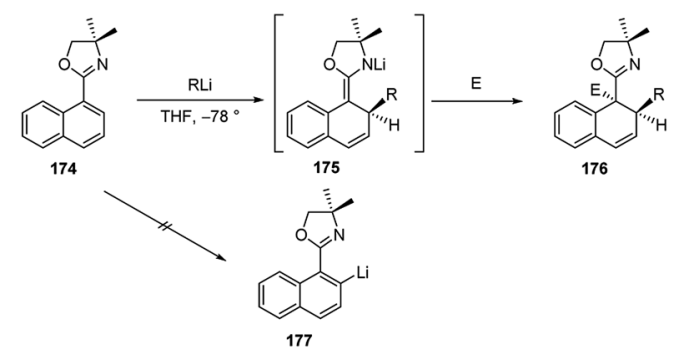

Scheme 58 Carbolithiation of naphtalene derivatives 174.

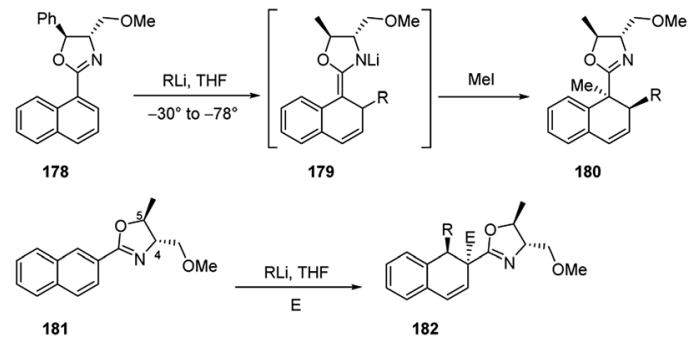

Scheme 59 Enantioselective carbolithiation of naphtalene oxazolines 178 and 181.

isocyanate, benzaldehyde, $\mathrm{Bu}_{3} \mathrm{SnCl}$, diethyl oxalate, and methyl formate were effective, as well as halogenating agents such as $\mathrm{CBr}_{4}, \mathrm{I}_{2}$, hexachloroethane, and $N$-fluorobenzenesulfonimide.

The integrated flow microreactor system was also applied to the synthesis of boscalid (173), an important fungicide agent (Scheme 57). Thus, the biaryllithium intermediate 170, generated as in Scheme 56, was reacted with tosyl azide providing $4^{\prime}$ chloro-2-azidobiphenyl 171a in $60 \%$ yield. Then, in batch, the reduction with $\mathrm{NaBH}_{4}$ to amine 172, followed by the reaction with 2-chloronicotinoyl chloride led to 173 in $88 \%$ yield (Scheme 57). ${ }^{72}$

\subsection{Naphthalene derivatives}

The addition of organolithiums to naphtalene derivatives was extensively developed by Meyers. ${ }^{73}$ During the investigation upon the $o$-metalation of 1-naphthyloxazoline $\mathbf{1 7 4}$ to provide the 2-naphthyllithium derivative 177, it was observed the conjugate addition of organometallics to such achiral naphthalene compound, leading to the intermediate $\mathbf{1 7 5}$. The subsequent electrophilic trapping led to the doubly alkylated product 176 in good yield (Scheme 58). The addition of alkyllwithium reagents onto the naphthalene $\pi$ system to give tandem 1,2-addition products similar to 175 had been observed 
earlier, with generally poor success. It was noted that the effect of the oxazoline substituent made this reaction considerably more effective ${ }^{74}$ than earlier efforts and opened up the way to a possible asymmetric version of this process.

Indeed, the asymmetric additions of alkyllithiums were successful both onto chiral 1- and 2-naphthyloxazolines 178 and 181 (Scheme 59). The addition step on $\mathbf{1 7 8}$ led to the formation of the intermediate azaenolate 179, which was trapped by various electrophilic reagents (e.g., MeI) at low temperatures providing the doubly alkylated dihydronaphthalene $\mathbf{1 8 0}$ in $>95$ : 5 diastereoisomeric ratios. The products were exclusively trans disubstituted ( $\mathrm{R}$ and $\mathrm{Me}$ ) and none of the cis isomers were detected. ${ }^{73}$ In the same way, the tandem addition-trapping reaction on 181 provided the trisubstituted dihydronaphthalenes 182 in good yields and high diastereomeric ratios. In this case, it was observed that the stereochemistry at the methoxymethyl groups (C-4) in the oxazolines was the major responsible for the stereochemical outcome of the alkyllithium addition. On the contrary, it was found that the stereochemistry and size $(\mathrm{Ph}, \mathrm{Me}, \mathrm{H})$ of the substituent at $\mathrm{C}-5$ on the oxazoline had very little effect on the stereochemical addition to the naphthalene $\pi$ system. $^{73}$

\section{Conclusions and outlook}

The inter-molecular carbolithiation of unfunctionalized and functionalized alkenes, when followed by electrophile trapping, provides a versatile and useful tool for the construction of complex molecular systems, allowing the simultaneous creation of up to two novel carbon-carbon or carbon-heteroatom bonds. By selecting proper substrates, additives, and reaction conditions, a high level of chemo-, regio-, and stereo-control can be achieved. Moreover, by employing chiral ligands, asymmetric processes can be obtained, even leading to enantioenriched quaternary stereocenters. However, despite the large number of examples reported, the synthetic potential of such process has not been completely explored yet. In particular, enantioselective processes have been developed for a limited number of substrates, always require stoichiometric or excess amounts of the chiral ligand, and only in a few cases have provided satisfactory enantiocontrol. For these reasons the synthetic application of the asymmetric inter-molecular carbolithiation is severely hampered. Moreover, the most popular and efficient chiral ligand used so far in these reactions, i.e. the natural alkaloid (-)-sparteine (L1), shows a strongly substrate dependent stereoselectivity and nowadays is no more easily commercially available. The discovery of more efficient chiral ligands for this reaction is then highly advisable. Besides the chiral diamine and diether ligands used in the reaction so far (Fig. 1), chiral 1,2-aminoethers look appealing candidates for developing novel and efficient chiral ligands. In fact, they have provided promising results in the enantioselective intramolecular carbolithiation, where chiral 1,2-aminoethers derived from pseudoephedrine or ephedrine ${ }^{75}$ and, more recently, from naturally occurring $\alpha$-amino or $\alpha$-hydroxy acids have been employed. ${ }^{76}$ In the latter example, both high enantioselectivity (up to $90 \%$ e.e.) and good substrate versatility in the intramolecular cyclization of aryl-allyl lithium derivatives were obtained. Moreover, in some cases, satisfactorily yields and enantioselectivity were obtained by employing substoichiometric ( 0.25 equiv.) amounts of the ligand. ${ }^{76}$ This result could open the way to the development of novel catalytic procedures, thus significantly enlarging the synthetic appeal of the reaction. A further, significant, improvement could be provided by the use in the carbolithiations of functionalized organolithiums, ${ }^{77-79}$ which would allow the construction of complex molecular systems. To this end, a promising scenario is provided by the recent advent of flow microreactor chemistry which allows the synthesis and manipulation of organolithiums functionalized even with electrophilic moieties. ${ }^{\mathbf{8 0}}$

\section{Conflicts of interest}

There are no conflicts to declare.

\section{Acknowledgements}

Financial support from Università della Basilicata (R.I.L. 2016 grant) and from MIUR, Project PON RI 2014-2020 BIOFEEDSTOCK (grant number ARS01_00985) is gratefully acknowledged.

\section{Notes and references}

1 P. Barbier, C. R. Hebd. Seances Acad. Sci., 1899, 128, 110-111.

2 V. Grignard, C. R. Hebd. Seances Acad. Sci., 1900, 130, 13221324.

3 I. Marek, J. Chem. Soc., Perkin Trans. 1, 1999, 535-544.

4 P. Knochel, Carbometallation of Alkenes and Alkynes in Comprehensive Organic Synthesis, ed. B. M. Trost, Pergamon, Oxford, 1991, vol. 4, pp. 867-872.

5 A.-M. L. Hogan and D. F. O'Shea, Chem. Commun., 2008, 3839-3851.

6 A. Gómez-SanJuan, N. Sotomayor and E. Lete, Beilstein J. Org. Chem., 2013, 9, 313-322.

7 C. Elschenbroich and J. Oliveira, Organometallics, WileyVCH, Weinheim, 3rd edn, 2003.

8 D. Baskara and A. H. Muller, Anionic Vinyl Polymerization in Controlled and living polymerizations: from mechanisms to applications, Wiley-VCH Verlag GmbH \& Co. KgaA, Weinheim, 2010.

9 Y. Minko and I. Marek, Advances in Carbolithiation in Lithium Compounds in Organic Synthesis, ed. R. Luisi and V. Capriati, Wiley-VCH Verlag GmbH \& Co. KGaA, Weinheim, Germany, 2014, pp. 329-350.

10 G. Eppe, D. Didier and I. Marek, Chem. Rev., 2015, 115, 91759206.

11 P. Beak and A. I. Meyers, Acc. Chem. Res., 1986, 19, 356-363. 12 M. C. Whisler, S. MacNeil, V. Snieckus and P. Beak, Angew. Chem., Int. Ed., 2004, 43, 2206-2225.

13 E. Carl and D. Stalke, Structure-Reactivity Relationship in Organolithium Compounds in Lithium Compounds in Organic Synthesis: From Fundamentals to Applications, ed. R. Luisi and V. Capriati, Wiley-VCH, Weinheim, 2014. 
14 D. M. Hodgson and M. A. H. Stent, Organolithiums in Enantioselective Synthesis, Springer, Verlag Berlin Heidelberg, 2003, vol.5.

15 J. Clayden, Organolithiums: selectivity for synthesis, Pergamon Press, Oxford, UK, 2002, pp. 258-259.

16 For a review on role of (-)-sparteine with organolithium compounds see: D. Hoppe and T. Hense, Angew. Chem., Int. Ed. Engl., 1997, 36, 2282-2316.

17 J. Clayden, Organolithiums: selectivity for synthesis, Pergamon Press, Oxford, UK, 2002, pp. 273-281.

18 D. R. Williams and J. T. Reeves, J. Am. Chem. Soc., 2004, 126, 3434-3435.

19 D. R. Williams, J. T. Reeves, P. P. Nag, W. H. Jr Pitcock and M.-H. Baik, J. Am. Chem. Soc., 2006, 128, 12339-12348.

20 S. Bywxter and D. J. Worspol, Can. J. Chem., 1962, 40, 15641575.

21 X. Wei and R. J. K. Taylor, J. Chem. Soc., Chem. Commun., 1996, 187-188.

22 X. Wei, P. Johnson and R. J. K. Taylor, J. Chem. Soc. Perkin Trans. I, 2000, 1109-1116.

23 X. Wei and R. J. K. Taylor, Tetrahedron: Asymmetry, 1997, 8, 665-668.

24 X. Wei and R. J. K. Taylor, Tetrahedron Lett., 1996, 37, 42094210.

25 S. Norsikian, I. Marek and J. F. Normant, Tetrahedron Lett., 1997, 38, 7523-7526.

26 S. Norsikian, I. Marek, S. Klein, J. F. Poisson and J. F. Normant, Chem.-Eur. J., 1999, 5, 2055-2068.

27 V. H. Gessner, S. G. Koller, C. Strohmann, A. M. Hogan and D. F. O'Shea, Chem.-Eur. J., 2011, 17, 2996-3004.

28 X. Wei and R. J. K. Taylor, Tetrahedron Lett., 1997, 38, 64676470.

29 X. Wei and R. J. K. Taylor, Tetrahedron Lett., 2003, 44, 71437146.

30 X. Wei and R. J. K. Taylor, Angew. Chem., Int. Ed., 2000, 39, 409-412.

31 C. M. Coleman and D. F. O'Shea,J. Am. Chem. Soc., 2003, 125, 4054-4055.

32 A. Kessler and C. M. Coleman, J. Org. Chem., 2004, 69, 78367846.

33 B. Cottineau and D. F. O'Shea, Tetrahedron, 2007, 63, 1035410362.

34 A. M. L. Hogan and D. F. O'Shea, J. Org. Chem., 2008, 73, 2503-2509.

35 A. M. L. Hogan and D. F. O'Shea, J. Am. Chem. Soc., 2006, 128, 10360-10361.

36 A. M. L. Hogan, T. Tricotet, A. Meek, S. S. Khokhar and D. F. O'Shea, J. Org. Chem., 2008, 73, 6041-6044.

37 A. M. L. Hogan and D. F. O'Shea, Org. Lett., 2006, 8, 37693772 .

38 A. M. L. Hogan and D. F. O'Shea, J. Org. Chem., 2007, 72, 9557-9571.

39 T. Kato, S. Marumoto, T. Sato and I. Kuwajima, Synlett, 1990, 671-672.

40 S. Klein, I. Marek and J.-F. Normant, J. Org. Chem., 1994, 59, 2925-2926.
41 C. Mück-Lichtenfeld and H. Ahlbrecht, Tetrahedron, 1999, 55, 2609-2624.

42 C. Mück-Lichtenfeld and H. Ahlbrecht, Tetrahedron, 1996, 52, 10025-10042.

43 S. Klein, I. Marek, J.-F. Poisson and J.-F. Normant, J. Am. Chem. Soc., 1995, 117, 8853-8854.

44 M. J. Dearden, M. J. McGrath and P. O'Brien, J. Org. Chem., 2004, 69, 5789-5792.

45 S. Norsikian, I. Marek, J.-F. Poisson and J.-F. Normant, J. Org. Chem., 1997, 62, 4898-4899.

46 S. Majumdar, A. de Meijere and I. Marek, Synlett, 2002, 3, 423-426.

47 N. Brémand, J.-F. Normant and P. Mangeney, Synlett, 2000, 4, 532-534.

48 N. Brémand, P. Mangeney and J.-F. Normant, Tetrahedron Lett., 2001, 42, 1883-1885.

49 S. Superchi, N. Sotomayor, G. Miao, B. Joseph, M. G. Campbell and V. Snieckus, Tetrahedron Lett., 1996, 37, 6061-6064.

50 J. G. Peters, M. Seppi, R. Fröhlich, B. Wibbeling and D. Hoppe, Synthesis, 2002, 3, 381-392.

51 A. M. Fournier and J. Clayden, Org. Lett., 2012, 14, 142-145. 52 J. Lefranc, A. Minassi and J. Clayden, Beilstein J. Org. Chem., 2013, 9, 628-632.

53 D. Castagnolo, D. J. Foley, H. Berber, R. Luisi and J. Clayden, Org. Lett., 2013, 15, 2116-2119.

54 D. Castagnolo, L. Degennaro, R. Luisi and J. Clayden, Org. Biomol. Chem., 2015, 13, 2330-2340.

55 F. Lepifre, B. Cottineau, D. Mousset, P. Bouyssou and G. Coudert, Tetrahedron Lett., 2004, 45, 483-484.

56 B. Cottineau, I. Gillaizeau, J. Farard, M.-L. Auclair and G. Coudert, Synlett, 2007, 12, 1925-1929.

57 T. K. Beng, H. Takeuchi, M. Weberc and R. Sarpong, Chem. Commun., 2015, 51, 7653-7656.

58 T. K. Beng, N. Fox, D. P. Bassler, A. Alwali, K. Sincavage and A. W. V. Silaire, Org. Biomol. Chem., 2015, 13, 8647-8651.

59 J. Yang and G. B. Dudley, Adv. Synth. Catal., 2010, 352, 34383442.

60 J. Clayden, M. Donnard, J. Lefranc, A. Minassi and D. J. Tetlow, J. Am. Chem. Soc., 2010, 132, 6624-6625.

61 M. Tait, M. Donnard, A. Minassi, J. Lefranc, B. Bechi, G. Carbone, P. O'Brien and J. Clayden, Org. Lett., 2013, 15, 34-37.

62 M. B. Tait, S. Butterworth and J. Clayden, Org. Lett., 2015, 17, 1236-1239.

63 M. Hojo, Y. Murakami, H. Aihara, R. Sakuragi, Y. Baba and A. Hosomi, Angew. Chem., Int. Ed., 2001, 40, 621-623.

64 K. Igawa and K. Tomooka, Angew. Chem., Int. Ed., 2006, 45, 232-234.

65 N. F. McKinley and D. F. O'Shea, J. Org. Chem., 2006, 71, 9552-9555.

66 D. Heijnen, M. van Zuijlen, F. Tosi and B. L. Feringa, Org. Biomol. Chem., 2019, 17, 2315-2320.

67 G. Babu, A. Orita and J. Otera, Chem. Lett., 2008, 37, 12961297.

68 E. Shirakawa, D. Ikeda, T. Ozawa, S. Watanabe and T. Hayashi, Chem. Commun., 2009, 1885-1887. 
69 J.-i. Yoshida, Basics of Flow Microreactor Synthesis, Springer Briefs in Molecular Science, Springer Japan, 2015, pp. 73-77.

70 Y. Tomida, A. Nagaki and J.-i. Yoshida, Org. Lett., 2009, 11, 3614-3617.

71 Y. Tomida, A. Nagaki and J.-i. Yoshida, J. Am. Chem. Soc., 2011, 133, 3744-3747.

72 A. Nagaki, D. Ichinari and J.-i. Yoshida, J. Am. Chem. Soc., 2014, 136, 12245-12248.

73 A. I. Meyers, J. Org. Chem., 2005, 70, 6137-6151.

74 A. I. Meyers, K. A. Lutomski and D. Laucher, Tetrahedron, 1988, 44, 3107-3118.
75 M. J. Mealy, M. R. Luderer, W. F. Bailey and M. B. Sommer, J. Org. Chem., 2004, 69, 6042-6049.

76 H. Guyon, A. Boussonnière and A.-S. Castanet, J. Org. Chem., 2017, 82, 4949-4957.

77 G. M. Dobrikov, I. Philipova, R. Nikolova, B. Shivachev, A. Chimov and V. Dimitrov, Polyhedron, 2012, 45, 126-143.

78 C. Nájera, J. M. Sansano and M. Yus, Tetrahedron, 2003, 59, 9255-9303.

79 C. Nájera and M. Yus, Curr. Org. Chem., 2003, 7, 867-926. 80 A. Nagaki, Tetrahedron Lett., 2019, 60, 150923. 